\title{
First simultaneous optical/near-infrared imaging of an X-ray selected, high-redshift cluster of galaxies with GROND
}

\section{The galaxy population of XMMU J0338.7+0030 at $z=1.1^{\star}$}

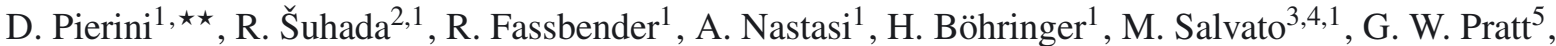 \\ M. Lerchster ${ }^{1, \star \star}$, P. Rosati ${ }^{6}$, J. S. Santos ${ }^{7}$, A. de Hoon ${ }^{8}$, J. Kohnert ${ }^{8}$, G. Lamer ${ }^{8}$, J. J. Mohr ${ }^{9,1,10}$ \\ M. Mühlegger ${ }^{1, \star \star}$, H. Quintana ${ }^{11}$, A. Schwope ${ }^{8}$, V. Biffi ${ }^{1}$, G. Chon ${ }^{1}$, S. Giodini ${ }^{12}$, J. Koppenhoefer ${ }^{1,2}$, \\ M. Verdugo ${ }^{1}$, F. Ziparo ${ }^{1}$, P. M. J. Afonso ${ }^{13,1}$, C. Clemens ${ }^{1, \star \star}$, J. Greiner ${ }^{1}$, T. Krühler ${ }^{14,10,1}$, A. Küpcü Yoldaş ${ }^{15}$, \\ F. Olivares E. ${ }^{1}$, A. Rossi ${ }^{16}$, and A. Yoldaş ${ }^{15}$ \\ (Affiliations can be found after the references)
}

Received 8 February 2011 / Accepted 13 December 2011

\begin{abstract}
Context. The XMM-Newton Distant Cluster Project is a serendipitous survey for clusters of galaxies at redshifts $z \geq 0.8$ based on deep archival $X M M$-Newton observations. X-ray sources identified as extended are screened against existing optical all-sky surveys for galaxies, in case of candidate high- $z$ clusters followed up with imaging at $4 \mathrm{~m}$-class telescopes and, ultimately, multi-object spectroscopy at $8 \mathrm{~m}$-class telescopes. Low-significance candidate high- $z$ clusters are followed up with the seven-channel imager GROND (Gamma-Ray Burst Optical and Near-Infrared Detector) that is mounted at a $2 \mathrm{~m}$-class telescope. Its unique capability of simultaneous imaging in the $g^{\prime}, r^{\prime}, i^{\prime}, z^{\prime}, J, H, K$ s bands enables the use of the photometric redshift technique.

Aims. Observing strategy, data reduction and analysis, depth and accuracy of the simultaneous multi-wavelength photometry are discussed with the goal of establishing GROND as a useful instrument to confirm X-ray selected (high- $z$ ) clusters.

Methods. The test case is XMMU J0338.7+0030, suggested to be at $z \sim 1.45 \pm 0.15(1 \sigma)$ from the analysis of the $z-H$ vs. $H$ colour-magnitude diagram obtained from the follow-up imaging. Later VLT-FORS2 spectroscopy enabled us to identify four members, which set this cluster at $z=1.097 \pm 0.002(1 \sigma)$. To reach a better knowledge of its galaxy population, we observed XMMU J0338.7+0030 with GROND for about $6 \mathrm{~h}$. The publicly available photo- $z$ code le Phare was used.

Results. The $K$ s-band number counts of the non-stellar sources out of the 832 detected down to $z^{\prime} \sim 26 \mathrm{AB}$ mag $(1 \sigma)$ in the $3.9 \times 4.3 \mathrm{arcmin}^{2}$ region of XMMU J0338.7+0030 imaged at all GROND bands clearly exceed those computed in deep fields/survey areas at $\sim 20.5-22.5$ AB mag. The photo- $z$ 's of the three imaged spectroscopic members yield $z=1.12 \pm 0.09(1 \sigma)$. The spatial distribution and the properties of the GROND sources with a photo- $z$ in the range 1.01-1.23 confirm the correspondence of the X-ray source with a galaxy over-density at a significance of at least $4.3 \sigma$. Candidate members that are spectro-photometrically classified as elliptical galaxies define a red locus in the $i^{\prime}-z^{\prime}$ vs. $z^{\prime}$ colour-magnitude diagram that is consistent with the red sequence of the cluster RDCS J0910+5422 at $z=1.106$. XMMU J0338.7+0030 hosts also a population of bluer late-type spirals and irregulars. The starbursts among the photometric members populate both loci, consistently with previous results.

Conclusions. The analysis of the available data set indicates that XMMU J0338.7+0030 is a low-mass cluster $\left(M_{200} \sim 10^{14} M_{\odot}\right)$ at $z=1.1$. With the photometric accuracy yielded by the present unoptimized multi-band observations with GROND, we not only confirm the spectroscopic redshift of this cluster but also show that it hosts a galaxy population that can still undergo significant bursts of star-formation activity.
\end{abstract}

Key words. X-rays: galaxies: clusters - galaxies: distances and redshifts - galaxies: clusters: general - galaxies: high-redshift

\section{Introduction}

A process of self-similar gravitational clustering in an expanding universe leads to the build-up of structures in a hierarchical fashion across time (White \& Rees 1978; White et al. 1987). Ensembles of galaxies and hot $\left(T \geq 10^{6} \mathrm{~K}\right)$ plasma within gravitationally bound haloes dominated by dark matter define groups and clusters, with total masses from $\sim 10^{13}$ to $\sim 10^{15} M_{\odot}$, respectively. These objects offer a biased view of the evolution of the large-scale structure (Kaiser 1984) that is easy to model theoretically from ab-initio principles.

The identification of a group/cluster requires observations at rest-frame optical/near-infrared (IR) wavelengths (where the

\footnotetext{
* Appendices are only available in electronic form at

http: //www. aanda. org

$\star \star$ Currently visiting astronomer at the MPE.
}

photospheric stellar emission dominates), in X-rays (where the hot plasma ${ }^{1}$ emits), or at sub-millimeter wavelengths (owing to the Sunyaev-Zel'dovich - SZ - effect, Sunyaev \& Zel'dovich 1972). In particular, the X-ray or SZ-effect detection ensures that a galaxy system is truly bound and associated with a deep gravitational potential well. X-ray observations of groups/clusters can now be pushed towards the distant universe (i.e., $z \geq 0.8$ ) thanks to the Chandra and XMM-Newton space-borne observatories (e.g., Stanford et al. 2001, 2002; Rosati et al. 2004; Mullis et al. 2005; Finoguenov et al. 2007; S̆uhada et al. 2010; however, see Rosati et al. 1998). With the SZ-effect this has become possible very recently but only for massive clusters (Staniszewski et al. 2009; Vanderlinde et al. 2010; Marriage et al. 2011).

\footnotetext{
${ }^{1}$ Whether the hot X-ray emitting plasma belongs to a group or a cluster, we will refer to it as the intracluster medium (ICM).
} 
Compiling a sample of distant galaxy clusters has a twofold importance. Firstly, it offers a way to trace structure growth in the far universe that enables the study of the effects of cold dark matter (CDM) and dark energy (DE) on the cosmic structure evolution (e.g., Albrecht et al. 2006; Vikhlinin et al. 2009; Mantz et al. 2010a). Secondly, it fosters the investigation of the evolution of the galaxy population, the baryon mass component of groups/clusters, the thermodynamics and chemical abundance of the ICM (e.g., Postman et al. 2005; Mei et al. 2006a,b; Balestra et al. 2007; Gobat et al. 2008; Maughan et al. 2008; Giodini et al. 2009; Mantz et al. 2010b; Santos et al. 2010; Strazzullo et al. 2010). About twenty clusters at $z=0.8-2.1$ have been discovered and studied in some detail (e.g., Stanford et al. 2001, 2002, 2006; Blakeslee et al. 2003; Mullis et al. 2005; Demarco et al. 2007; Hilton et al. 2007, 2009; Eisenhardt et al. 2008; Lamer et al. 2008; Menci et al. 2008; Wilson et al. 2009; Kurk et al. 2009; Tanaka et al. 2010; Papovich et al. 2010; Henry et al. 2010; Gobat et al. 2011; Fassbender et al. 2011a). However, a better sampling in redshift and total mass is necessary to build a representative sample of high- $z$ groups and clusters. Existing $\mathrm{X}$-ray surveys allow clusters in the redshift range $0.8-1.5$ to be detected, with about 1.5 such clusters per square degree down to a flux level of $\sim 6 \times 10^{-15} \mathrm{erg} \mathrm{s}^{-1} \mathrm{~cm}^{-2}$ (S̆uhada et al. 2012).

The XMM-Newton Distant Cluster Project (XDCP) is a serendipitous survey for galaxy clusters at $z \geq 0.8$ based on deep archival XMM-Newton observations: it now covers an area of $80 \mathrm{deg}^{2}$ (Böhringer et al. 2005; Fassbender 2007; Fassbender et al. 2011c). XDCP is concerned with the buildup of a representative and complete sample of distant clusters and the accurate determination of the total mass of each system. Both aspects are crucial for a test of the current understanding of structure formation and evolution in $\Lambda \mathrm{CDM}$ universes (e.g., Hoyle et al. 2011). In XDCP, X-ray sources identified as extended are screened against existing optical all-sky surveys and eventually followed up with imaging in the $z, H$ or $I, J$ bands at 4 m-class telescopes (occasionally in the $r, Z$ bands at $8 \mathrm{~m}$-class telescopes). If the distribution of the galaxies associated with an X-ray source exhibits evidence of a so-called "red sequence" (e.g. Bower et al. 1992; Kodama et al. 1998; Gladders \& Yee 2000; see Arimoto \& Yoshii 1987, for its physical interpretation) in the ensuing colour-magnitude diagram, a candidate high- $z$ cluster is robustly selected and proposed for spectroscopic confirmation at $8 \mathrm{~m}$-class telescopes. This strategy has already yielded more than 30 spectroscopically confirmed distant clusters since the discovery of XMMU J2235.3-2557 at $z=$ 1.393 (Mullis et al. 2005). They include XMMU J0338.8+0021 at $z=1.49$ (Nastasi et al. 2011), XMMU J1007.4+1237 at $z=1.555$ (Fassbender et al. 2011b) and XMMU J0044.0-2033 at $z=1.579$ (Santos et al. 2011).

Among the main results from XDCP we quote the multiwavelength studies of XMMU J1229+0151 at $z=0.975$ (Santos et al. 2009) and XMMU J2235.3-2557 (Rosati et al. 2009; Strazzullo et al. 2010); the weak-lensing estimate of the total mass of XMMU J2235.3-2557 (Jee et al. 2009); the total mass estimate of XMMU J100750.5+125818 ( $z=1.082)$ from strong lensing, optical spectroscopy and X-ray imaging (Schwope et al. 2010); the pan-chromatic study of XMMU J1230.3+1339 at $z=0.975$ (Fassbender et al. 2011a) and its total mass estimate from weak-lensing analysis of ground-based imaging (Lerchster et al. 2010); the evidence of starburst activity in the core of XMMU J1007.4+1237 (Fassbender et al. 2011b).

In order to maximize the numerical throughput from XDCP, low-significance candidate high- $z$ clusters are followed up with the seven-channel imager GROND (Gamma-Ray Burst Optical and Near-Infrared Detector; Greiner et al. 2008), that is mounted at a $2 \mathrm{~m}$-class telescope, before being proposed for multi-object spectroscopy (MOS) at $8 \mathrm{~m}$-class telescopes. The optical confirmation of these distant clusters lies in the discovery of a significant feature in the distribution of the photometric redshifts of galaxies selected in a region corresponding to the extended $\mathrm{X}$-ray emission. Otherwise, it relies upon the fact that colouror photo-z-selected galaxies within a given projected distance from the centroid of the extended X-ray emission define an over-density with respect to the surrounding region and/or a red sequence in a given colour-magnitude diagram.

Here we report on the feasibility of this approach by illustrating results obtained from the multi-wavelength observations of XMMU J0338.7+0030. Follow-up $z, H$ photometry was originally obtained with the near-IR, wide-field camera OMEGA2000 mounted at the prime focus of the $3.5 \mathrm{~m}$ telescope at Calar Alto, Spain. A redshift $z \sim 1.45 \pm 0.15$ was inferred from comparison between the average $z-H$ colour of the reddest galaxies within $45^{\prime \prime}$ from the X-ray position of XMMU J0338.7+0030 and the synthetic $z-H$ colours expected for a large suite of simple stellar population (SSP) models (see Fassbender et al. 2011c). The synthetic photometry was computed using the spectro-photometric evolutionary synthesis model PÉGASE v2 (Fioc \& Rocca-Volmerange 1997) in analogy with Pierini et al. (2005) and Wilman et al. (2008). A calibration of the best-performing SSP model was originally based on observed red-sequence galaxies in $10 \mathrm{X}$-ray selected clusters with spectroscopic redshifts up to 1.5 (Fassbender 2007). This fiducial model corresponds to a Salpeter (1955) stellar initial mass function (IMF) between 0.1 and $120 M_{\odot}$ (as all the others), a solar metallicity and a formation redshift equal to 5 .

In addition to its being potentially one of the few clusters at $z \geq 1.4$ known so far, XMMU J0338.7+0030 represented a test case for XDCP sources at the detection limit of the survey. It was an ideal target for GROND imaging because its region is covered by the Sloan Digital Sky Survey (SDSS; York et al. 2000) and the 2 Micron All Sky Survey (2MASS; Skrutskie et al. 2006), which enables a robust photometric calibration for all seven bands. For the same region, sparse redshift information was later available from observations with the spectrograph FORS2 mounted at the ESO Very Large Telescope (VLT) at Paranal, Chile.

The XMM-Newton, OMEGA2000, GROND and FORS2 observations of XMMUJ0338.7+0030, plus the reduction and analysis of the ensuing data, are discussed in Sect. 2. Estimates of the X-ray bolometric luminosity, temperature and total mass of this cluster, plus the spectroscopic determination of its redshift, are given there. Section 3 contains results obtained from the GROND data, i.e. galaxy number counts, the photo- $z$ distribution of the detected sources, the two-dimensional (2-D) spatial distribution of photo- $z$-selected galaxies and their distribution in specific colour-magnitude diagrams. Discussion and conclusions appear in Sects. 4 and 5, respectively.

Throughout we adopt a $\Lambda$ CDM cosmological model $\left(\Omega_{\mathrm{m}}=\right.$ $\left.0.3, \Omega_{\Lambda}=0.7\right)$ with $H_{0}=70 \mathrm{~km} \mathrm{~s}^{-1} \mathrm{Mpc}^{-1}$, that is broadly consistent with the main results from the $5 \mathrm{yr}$ operations of the Wilkinson Microwave Anisotropy Probe (WMAP5, Dunkley et al. 2009; Komatsu et al. 2009). Accordingly, at the spectroscopic redshift of XMMU J0338.7+0030 $(z=1.097$; Sect. 2.4.2), the age of the Universe is $5.4 \mathrm{Gyr}$, the cosmic evolution factor $E(z)=H(z) / H_{0}$ is equal to 1.86 , the luminosity distance is $7409.2 \mathrm{Mpc}$, the angular scale is $8.168 \mathrm{kpc} /{ }^{\prime \prime}$. Hence, an angular distance of $1^{\prime}$ corresponds to $\sim 490 \mathrm{kpc}$ at the cluster distance. 


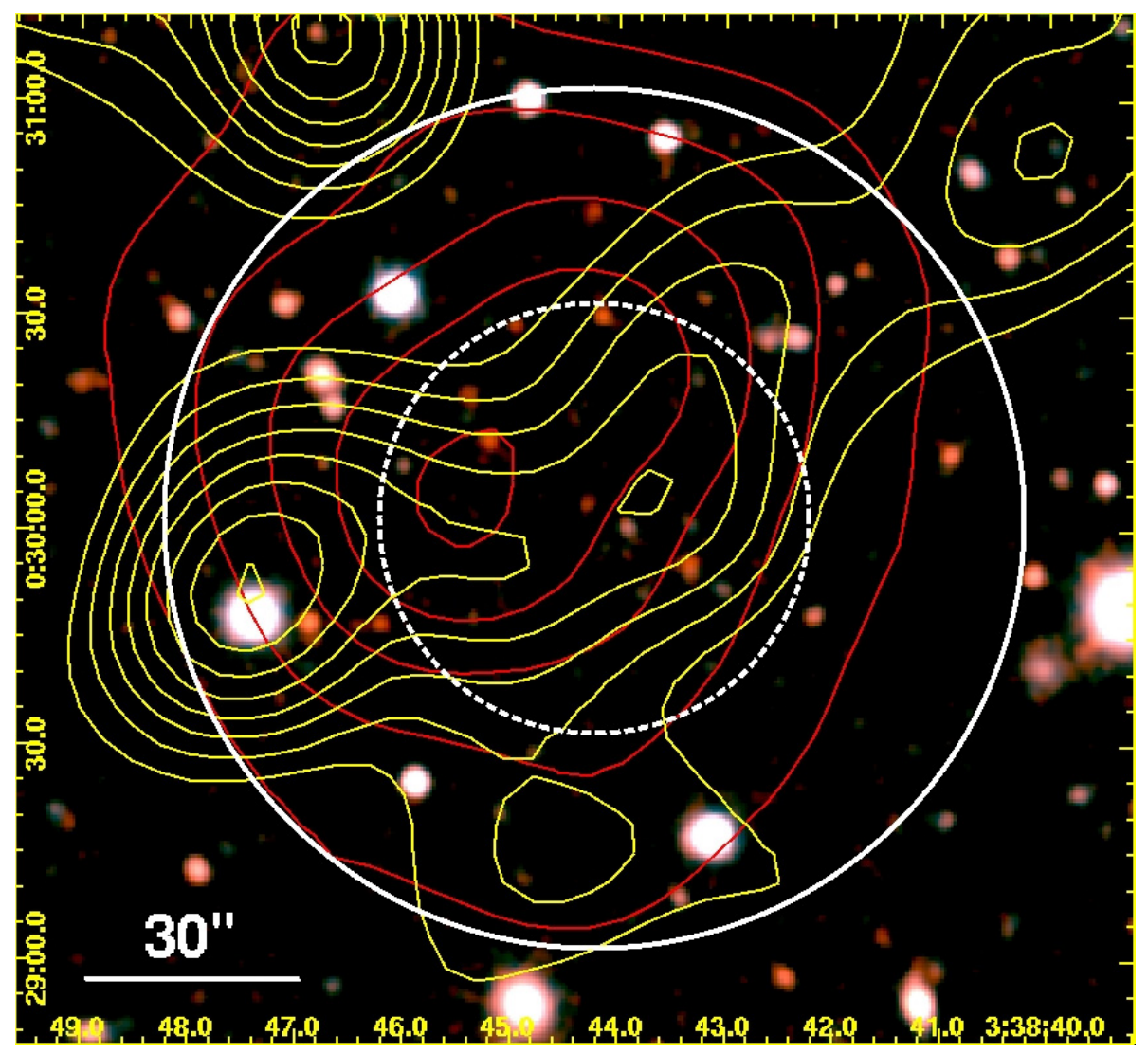

Fig. 1. A $z+H$ colour composite image $\left(4.5^{\prime}\right.$ on the side) of the environment of XMMU J0338.7+0030 with an overlaid contour map of the X-ray emission detected by $X M M-N e w t o n$ (in yellow). These log-spaced $\mathrm{X}$-ray contours correspond to significance levels of $2-16 \sigma$; they were derived from the adaptively smoothed, combined images of the cluster environment. Red contours highlight the over-density of galaxies with very red $z$ $H$ colours $(3.0 \leq z-H \leq 4.8$ Vega mag), as obtained from the OMEGA2000 imaging (see Sect. 2.2.2). White dashed and solid lines encircle regions within $30^{\prime \prime}$ and $60^{\prime \prime}$ from the $\mathrm{X}$-ray centroid of the cluster $(\mathrm{RA}(\mathrm{J} 2000.0)$ : $03^{\mathrm{h}} 38^{\mathrm{m}} 44.2^{\mathrm{s}}, \quad \operatorname{Dec}(\mathrm{J} 2000.0)$ : $\left.+00^{\mathrm{d}} 30^{\mathrm{m}} 01.8^{\mathrm{s}}\right)$, respectively; they guide the eye. The red contour closest to the X-ray position of the cluster corresponds to a significance of $4.6 \sigma$ above the mean background.

\section{Observations, data reduction and analysis}

\subsection{X-ray imaging with XMM-Newton}

\subsubsection{Initial X-ray selection}

A total of 470 XMM-Newton (Jansen et al. 2001) archival fields with nominal exposure times longer than $10 \mathrm{ks}$ have so far been processed and analyzed in XDCP as extensively described in Fassbender (2007) and Fassbender et al. (2011c). The serendipitous, weak X-ray source identified as XMMU J0338.7+0030 was detected in the field with observation identification number (OBSID) 0036540101. The target was SDSS 033829.31+002156.3 (RA(J2000.0): $03^{\mathrm{h}} 38^{\mathrm{m}} 38.5^{\mathrm{s}}$, $\operatorname{Dec}(\mathrm{J} 2000.0):+00^{\mathrm{d}} 39^{\mathrm{m}} 28^{\mathrm{s}}$ ), a quasar at $z=5.07091$ (Péroux et al. 2001) imaged on February 22, 2002 with XMM-Newton for a nominal exposure time of $22.9 \mathrm{ks}$.

This serendipitous X-ray source was found at an offaxis angle of $9.6^{\prime}\left(\mathrm{RA}(\mathrm{J} 2000.0)\right.$ : $03^{\mathrm{h}} 38^{\mathrm{m}} 44.2^{\mathrm{s}}, \operatorname{Dec}(\mathrm{J} 2000.0)$ : $\left.+00^{\mathrm{d}} 30^{\mathrm{m}} 01.8^{\mathrm{s}}\right)$ and a significance of about $5 \sigma$ during the early XDCP source detection run performed with SAS v6.1. The tasks eboxdetect was used for a sliding box detection, followed by a subsequent maximum likelihood analysis with emldetect. This yielded an extent of 3.48 pixels (i.e., 13.92 arcsec), a source extent likelihood of about $2 \sigma$ and a flux of $\sim 8 \times 10^{-15} \mathrm{erg} \mathrm{s}^{-1} \mathrm{~cm}^{-2}$ for $\sim 32 \mathrm{PN}$ counts, close to the detection threshold. Hence, XMMU J0338.7+0030 appeared as a marginally extended X-ray source at the XMM-Newton detection threshold for extended sources. An X-ray contour map is overlaid on the $z+H$ colour composite image obtained from the medium-deep imaging with OMEGA2000 (Sect. 2.2.1) in Fig. 1.

The circular area centred on the X-ray emission centroid of XMMU J0338.7+0030 and delimited by a radius of $1^{\prime}$ is assumed as the "bona fide" cluster region throughout.

\subsubsection{The upgraded $X$-ray picture of $X M M U J 0338.7+0030$}

The XMM-Newton field containing XMMU J0338.7+0030 was reprocessed with SAS v10.0.0 following Suhada et al. (2011). These observations are strongly flared and an automatic flare removal is impossible; hence, the contaminated periods were excised manually and the automatic two-step flare cleaning process was run on the remaining good part of the data for the removal of high background periods. The loss of information is severe: clean net exposure times on axis amount to $9 \mathrm{ks}$ for either MOS camera and $7.8 \mathrm{ks}$ for the PN instrument, whereas the effective (vignetted) clean net exposure times on source amount to $4.5-5.5 \mathrm{ks}$ for all detectors. Even after cleaning a slight residual quiescent contamination (about $10 \%$ above normal) remains; large part of it should be captured by the background model, so this anomalous contamination likely has a negligible effect.

With SAS v10.0.0 XMMUJ0338.7+0030 is detected as a very weak source without a significant extent, located about $10^{\prime \prime}$ to the east with respect to the X-ray position originally estimated (Sect. 2.1.1). A Kolmogorov-Smirnov (KS) test with the null hypothesis that the extracted X-ray profile corresponds to that of a point-like source gives $-\log P_{\mathrm{KS}}=2.08$ and $-\log P_{\mathrm{KS}}=1.57$ for the PN and MOS data, respectively. In other words, there is a chance of $0.8 \%$ or $2.7 \%$ that XMMU J0338.7+0030 is a point-like source. The result based on the PN instrument is likely unrealistic, because PN has a chip gap very close to the centre. Furthermore, the performed test uses the on-axis pointspread-function (PSF) of XMM-Newton, so the output probabilities are slightly underestimated for the off-axis source identified as XMMU J0338.7+0030. We conclude that this source is weak and marginally extended.

This is supported by the regular behaviour of the growth curves, where the growth curve analysis (GCA) method of Böhringer et al. (2000) was applied to measure the flux in the 


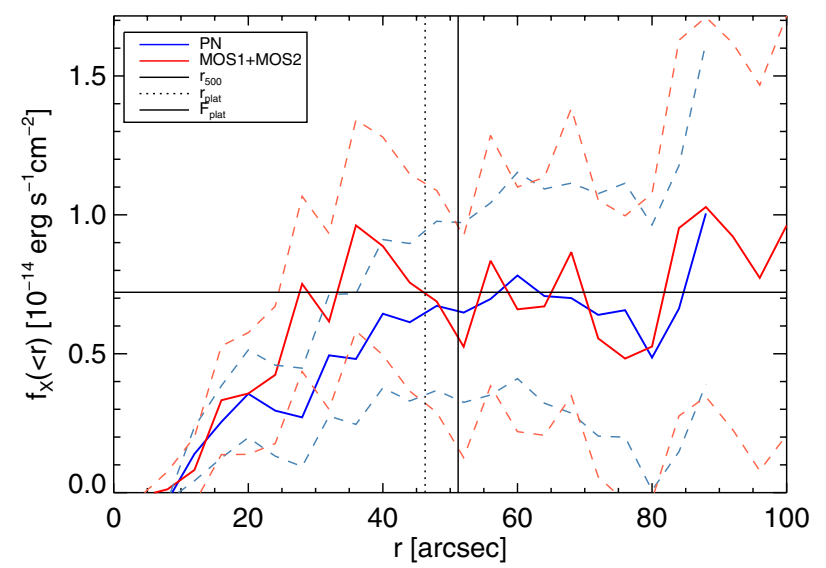

Fig. 2. Results from the application of the GCA method of Böhringer et al. (2000) in the soft $0.5-2 \mathrm{keV}$ band to the MOS and PN observations of XMMU J0338.7+0030. The two growth curves are well behaved and agree with each other.

soft $0.5-2 \mathrm{keV}$ band. The two growth curves obtained with the MOS cameras or the PN instrument agree (see Fig. 2). However, they are not independent: the latter was slightly corrected to obtain a rough agreement, because $\mathrm{PN}$ has a chip gap very close to the centre as already mentioned. The double component background model was used in the first instance; the alternative use of a spline fitting led to the same results. As a result, we measured a flux $f_{\mathrm{X}, 500} \simeq 7.1( \pm 2.3) \times 10^{-15} \mathrm{erg} \mathrm{s}^{-1} \mathrm{~cm}^{-2}$ within a circular aperture of radius equal to $R_{500}{ }^{2}$ (corresponding to $51.2^{\prime \prime}$ ). At the cluster redshift, this translates into an X-ray luminosity of $L_{\mathrm{X}, 500} \simeq 4.5( \pm 1.4) \times 10^{43} \mathrm{erg} \mathrm{s}^{-1}$ in the soft band or $L_{\mathrm{X}, 500}^{\mathrm{bol}} \simeq 1.1( \pm 0.3) \times 10^{44} \mathrm{erg} \mathrm{s}^{-1}$ for the bolometric luminosity. The latter quantity was inferred as described in Suhada et al. (2010). In brief, the growth curve was used to iteratively obtain a self-consistent set of parameters using the X-ray luminositytemperature and X-ray luminosity-total mass relations from Pratt et al. (2009).

Owing to the faintness of the source with less than 50 net counts in the soft band, the determination of additional structural or spectral parameters is currently not feasible. The potential flux contribution of point sources can presently not be accounted for either, which implies that the stated luminosities have to be taken as upper limits. However, based on the luminosity scaling relation in Pratt et al. (2009), first rough estimates on the expected temperature of $T_{\mathrm{X}} \sim 2.4 \mathrm{keV}$ and the total mass of $M_{500} \sim 7 \times 10^{13} M_{\odot}$ (i.e., $M_{200} \sim 10^{14} M_{\odot}$ ) can be obtained from the previous values. Hence, XMMU J0338.7+0030 appears to be a system at the boundary between X-ray selected groups and clusters, as XMMU J1532.2-0836 at $z=1.358$ (Šuhada et al. 2011) and XMMU J0338.8+0021 at $z=1.49$ (Nastasi et al. 2011).

An exposure-corrected count image of the innermost region of the cluster is produced after masking point-like sources (cf. Fig. 1) and is then adaptively smoothed with a circular top hat filter. The ensuing X-ray contours are reproduced in the right panel of Fig. 4 (Sect. 2.3.1) using a squared scale.

\footnotetext{
${ }^{2} R_{\Delta}(\Delta=500)$ is the radius within which the total mass density of a group/cluster is equal to $\Delta$ times the critical density of the universe $\left(\rho_{\mathrm{c}}\right)$. Correspondingly, $M_{\Delta}=\Delta \rho_{\mathrm{c}}(z)(4 \pi / 3) R_{\Delta}^{3}$ is the total mass within $R_{\Delta}$.
}

\subsection{Near-infrared follow-up imaging with OMEGA2000}

\subsubsection{Observations and data reduction}

XMMU J0338.7+0030 was observed with the near-IR camera OMEGA2000 (Bailer-Jones et al. 2000) that is mounted at the $3.5 \mathrm{~m}$ telescope at the Centro Astronómico Hispano Alemán (CAHA) at Calar Alto, Spain. OMEGA2000 provides a field-ofview $(\mathrm{FoV})$ of $15.4^{\prime} \times 15.4^{\prime}$. In particular, medium-deep imaging of the XMMU J0338.7+0030 region in the $H$-band ( $50 \mathrm{~min}$ ) and $z$-band (53 min) was obtained on January 5, 2006 under good but non-photometric conditions of the sky and with a seeing (or PSF) that varied between 1.0" and $1.5^{\prime \prime}$ ( $z$-band) or $1.6^{\prime \prime}$ ( $H$-band) in full-width-at-half-maximum (FWHM). The same region was reobserved in $z$-band (5 min) on October 30, 2006 in photometric conditions of the sky. Observations of a designated SDSS standard star (Smith et al. 2002) were performed then to enable a photometric calibration of the science frames.

These data were reduced with the OMEGA2000 near-IR pipeline (Fassbender 2007), which performs the basic frame calibrations (flat-fielding, bad pixel correction), a two-pass background subtraction with masked objects, frame alignment and optimally weighted co-addition of the individual $40 \mathrm{~s}$ (60 s) exposures in $H$-band ( $z$-band). For each band, the individual frames were stacked without PSF-matching, which gave a measured seeing of $1.3^{\prime \prime}$ ( $z$-band) or $1.4^{\prime \prime}(H$-band) in the final image.

For the CAHA data, source detection, extraction of photometry and star/galaxy separation are described in detail in Fassbdender et al. (2007). The SExtractor (Bertin \& Arnouts 1996) photometry was calibrated to the Vega magnitude system using 2MASS point sources (Cutri et al. 2003) in $H$-band and designated SDSS standard star observations in $z$-band, crosschecked with SDSS photometry in the science frame. In particular, total magnitudes were measured in a standard Kron (1980) aperture of factor 2.5 and minimum radius of 3.5. The limiting (Vega) magnitudes ${ }^{3}$ were determined to be $H_{\text {lim }} \sim 21.2$ and $z_{\lim } \sim 23.1$. They correspond to the expected apparent magnitudes of an old, passively evolving galaxy at the estimated redshift of $z \sim 1.45$ (see Sect. 1) for an absolute magnitude equal to $\sim M^{\star}+1.3$ in $H$-band and $\sim M^{\star}$ in $z$-band, where $M^{\star}$ is the characteristic magnitude of the corresponding galaxy luminosity function at $z=1.45$ (see Fassbender 2007).

\subsubsection{An over-density of very red galaxy at the location of XMMU J0338.7+0030}

In Fig. 1 (Sect. 2.1.1), the projected distribution of galaxies with very red colours ( $3.0 \leq z-H \leq 4.8$ Vega mag) exhibits a significant over-density: $4.6 \sigma$ above the mean background. The peak of this over-density is offest to the East by about $20^{\prime \prime}$ with respect to the X-ray position of XMMU J0338.7+0030. When considering the degradation of the angular resolution of XMM-Newton $\left(\sim 14^{\prime \prime}\right.$ half-energy-width - HEW) at the off-axis angle of this X-ray source (Sect. 2.1.1), an association between the previous over-density of red galaxies and the cluster is very likely.

Figure 3 shows the $z-H$ vs. $H$ colour-magnitude diagram for the galaxies detected in the XMMU J0338.7+0030 region imaged with OMEGA2000. In particular, seven galaxies within $45^{\prime \prime}$ from the X-ray position of the cluster exhibit $2.8 \leq$ $z-H \leq 3.8$ Vega mag). A redshift $z \sim 1.45 \pm 0.15$ was initially

3 These limiting magnitudes correspond to a 50\% completeness, as estimated from the number counts obtained from the OMEGA2000 imaging only, as in Fassbender et al. (2011a). 


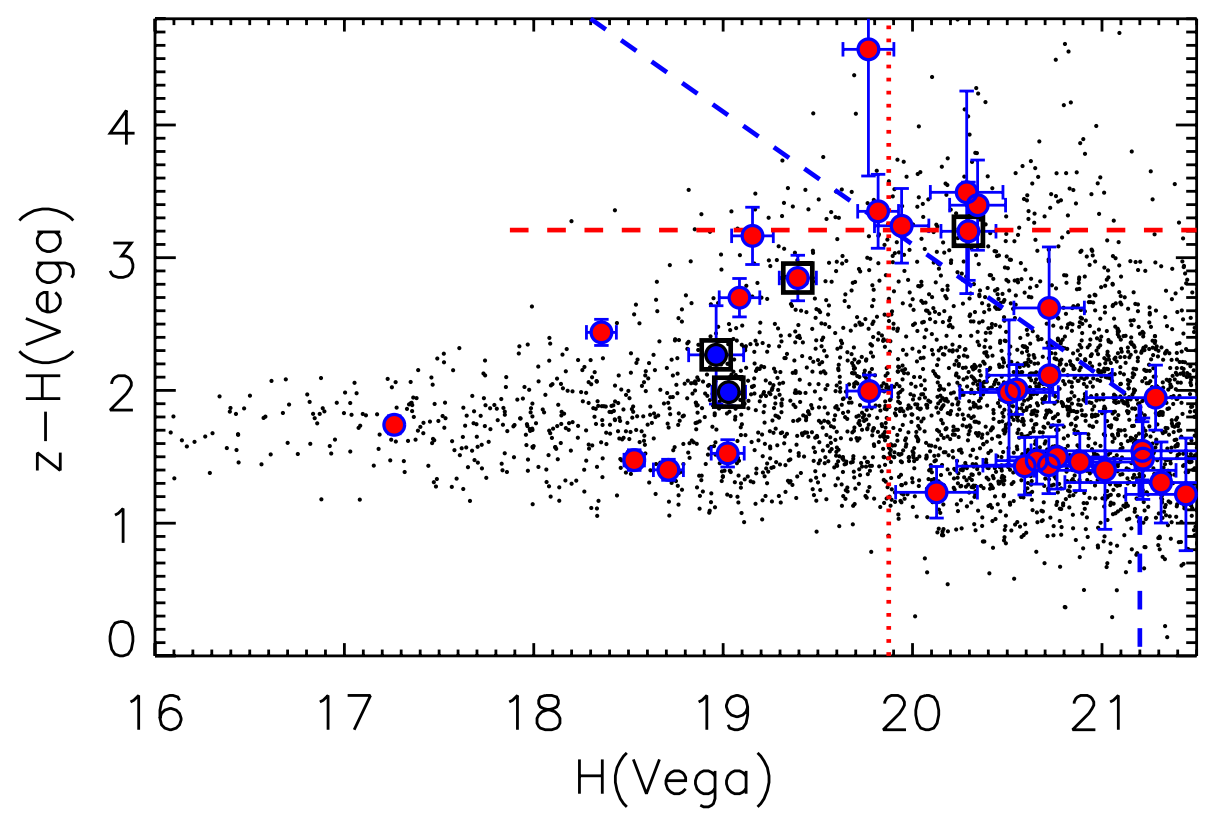

Fig. 3. $z-H$ vs. $H$ colour-magnitude diagram for the region encompassing XMMU J0338.7+0030 imaged with OMEGA2000. Red filled circles represent galaxies within $45^{\prime \prime}$ from the X-ray position of the cluster. The blue dashed lines reproduce the 50\% completeness of the photometric catalogue extracted from the OMEGA2000 data. The horizontal red short-dashed line represents the $z-H$ colour expected for an SSP model corresponding to the evolutionary phase of an instantaneous burst of star formation observed at $z=5$ with Salpeter (1955) stellar IMF and solar metallicity observed at $z=1.45$. This SSP model describes the average colour of the seven galaxies associated with XMMU J0338.7+0030 that exhibit $2.8 \leq z-H \leq 3.8$ Vega mag. The vertical red dotted line represents the characteristic magnitude $H^{\star}$ at $z=1.45$ (from Fassbender et al. 2007). Empty black squares mark the four spectroscopic cluster members that were successively established to be at $z=1.1$ through VLT-FORS2 observations (see Fig. 11 and Table 1 in Sect. 2.4.2); blue filled circles highlight those with ID = 12 and ID = 15, which are at a cluster-centric distance greater than $45^{\prime \prime}$. The two spectroscopic cluster members without convincing evidence of ongoing star-formation activity (with ID $=2$ and ID = 16) exhibit about as red $z-H$ colours as the previous SSP model (see Appendix B, however).

Table 1. Summary of the main properties of the four galaxies observed with OMEGA2000 and FORS2 identified as spectroscopic cluster members.

\begin{tabular}{lccccccc}
\hline \hline ID & $\begin{array}{c}\text { RA (J2000) } \\
\text { hh mm ss }\end{array}$ & $\begin{array}{c}\text { Dec (J2000) } \\
\text { dd mm ss }\end{array}$ & $\begin{array}{c}H \\
\text { Vega mag }\end{array}$ & $\begin{array}{c}z-H \\
\text { Vega mag }\end{array}$ & $z$ & D4000 & $\begin{array}{c}\text { [OII] } E W \\
\AA\end{array}$ \\
\hline 2 & 033844.136 & +003029.95 & 19.39 & 2.84 & $1.09437 \pm 0.00090$ & $1.901 \pm 0.052$ & \\
12 & 033843.026 & +003218.55 & 19.03 & 1.98 & $1.09787 \pm 0.00040$ & $1.194 \pm 0.036$ & $-32.908 \pm 1.380$ \\
15 & 033840.912 & +003010.66 & 18.96 & 2.27 & $1.09641 \pm 0.00027$ & $1.633 \pm 0.028$ & $-7.952 \pm 0.962$ \\
16 & 033844.299 & +003003.71 & 20.29 & 3.20 & $1.09930 \pm 0.00044$ & $1.690 \pm 0.131$ & $-9.291 \pm 3.873$ \\
\hline
\end{tabular}

inferred from comparison between the average $z-H$ colour of these galaxies and the synthetic $z-H$ colours expected for the fiducial SSP model described in Sect. 1. The assumption was that such a template described all those galaxies.

\subsection{Optical/near-infrared imaging with GROND}

\subsubsection{Simultaneous images of XMMU J0338.7+0030}

A region of the sky centred on the XMM-Newton X-ray position of XMMU J0338.7+0030 was imaged on four different nights (September 24, 2008, and January 27, 28, 29, 2009), in conditions of clear sky but variable seeing, with GROND. This is a seven-channel optical/near-IR imager primarily designed for rapid, simultaneous multi-wavelength observations of gammaray burst afterglows and built by the high-energy group of MPE in collaboration with the Landessternwarte Tautenburg and ESO. GROND was commissioned at the MPI/ESO $2.2 \mathrm{~m}$ telescope at La Silla, Chile, in April 2007 and is operated as a PI-instrument (for its comprehensive description see Greiner et al. 2008).

For the optical channels, the incoming telescope beam is split using four dichroics designed in such a way that their transmission functions are identical to those of the Sloan $g^{\prime}, r^{\prime}, i^{\prime}, z^{\prime}$ filter system, with the exception of the $i^{\prime}$-band. Each of the four identical optical CCDs has a FoV of $5.4 \times$ $5.4 \operatorname{arcmin}^{2}$ and a scale of $0.158^{\prime \prime} /$ pixel. The near-IR part of GROND is designed as a focal reducer system yielding a FoV of $10 \times 10 \mathrm{arcmin}^{2}$ with a scale of $0.6^{\prime \prime} /$ pixel. The GROND $J, H, K$ s dichroic filters are close to other near-IR filter systems. Photometric system transformations between the GROND $g^{\prime}, r^{\prime}, i^{\prime}, z^{\prime}$ bands and the Sloan filter system and between the GROND $J, H, K$ s bands and the 2MASS filter system are given in Greiner et al. (2008).

A total of 10 observations in the seven channels was executed through the GROND standard observing block (OB) named "20min4TD". By design, this OB corresponds to four single 369 s-long exposures in the $g^{\prime}, r^{\prime}$ channels and four 369 s-long exposures out of four pairs of sub-exposures in the $i^{\prime}, z^{\prime}$ channels, each long exposure being taken at one out of four different telescope dither positions. In parallel, the three near-IR channels are operated with $10 \mathrm{~s}$ integrations. At each telescope dither position, a six-position dither pattern is executed through a flip mirror (internal dithering) only for the $K$ s channel; five 10 s-long exposures per mirror position are executed 

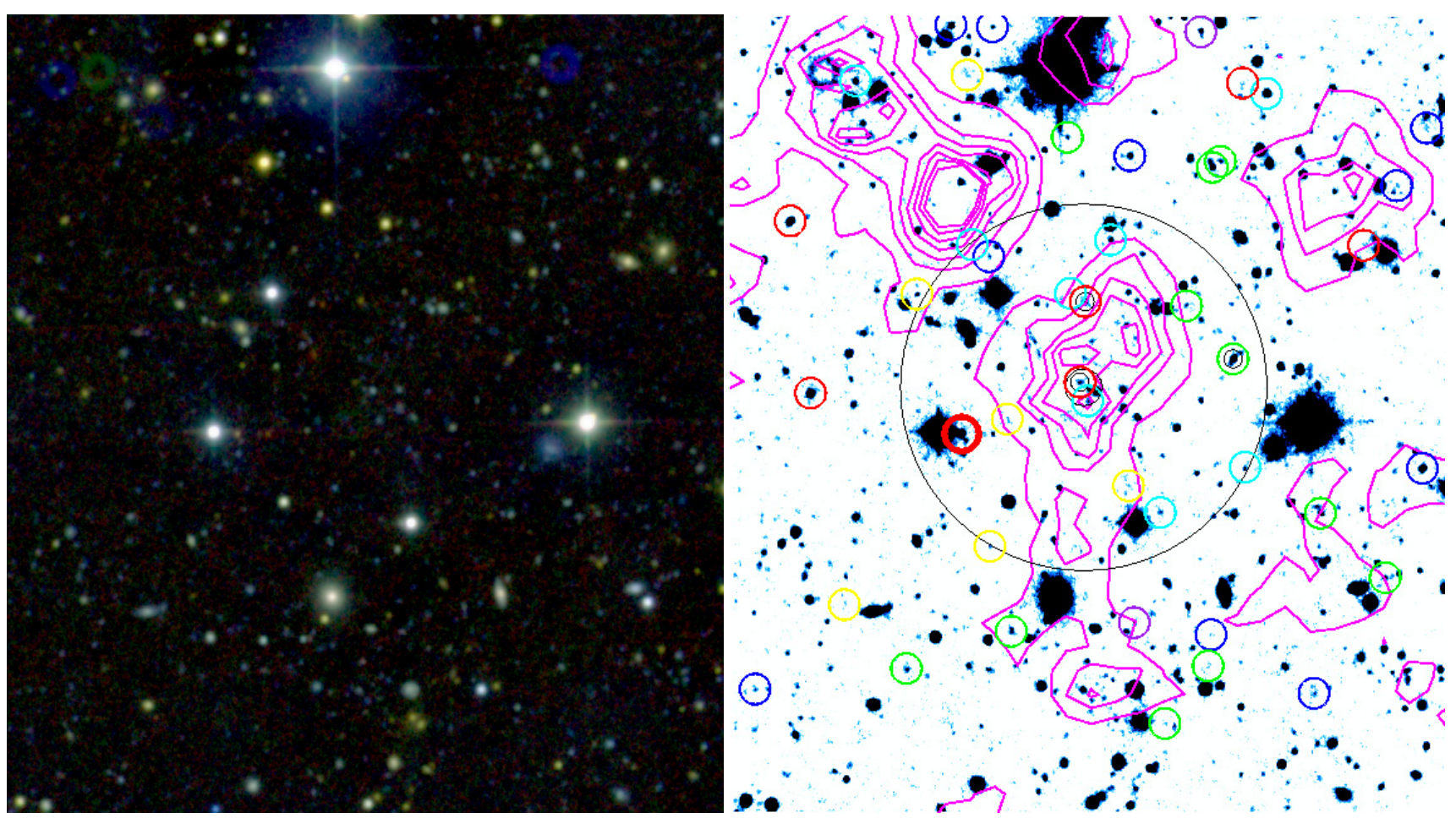

Fig. 4. $J, z^{\prime}, g^{\prime}$ colour-composite image of the $3.9 \times 4.3 \operatorname{arcmin}^{2}$ region of XMMU J0338.7+0030 mapped with GROND at all seven bands (left panel). North is up and east to the left. The corresponding $z^{\prime}$-image (right panel) illustrates the 2-D distribution of the 44 sources in the GROND photometric sample with a photo- $z$ that is consistent with the spectroscopic redshift, i.e., at $1.01 \leq z \leq 1.23$ (empty circles). These 44 photometric members are colour-coded according to their spectro-photometric classification as E (red), Sbc (yellow), Scd (green), Im (cyan), SB (blue) or QSO (purple). The three spectroscopic members among them are marked with small, black empty circles. Two concentric black circles with radii of 6" and 60" mark the X-ray position and bona fide region of XMMU J0338.7+0030, respectively. Magenta contours represent the weak, marginally extended X-ray emission of the source determined in Sect. 2.1.2. This emission is consistent with the projected distribution of the photometric cluster members. However, the candidate BCG (thick red empty circle) is offset by $\sim 43^{\prime \prime}$ towards the east-south-east.

for the $J, H, K$ s channels. This produces a total of 120 single 10 s-long exposures per OB for each near-IR channel. Given the impact of overheads (about $50 \%$ of the total exposure time of $4 \mathrm{~h}$ for each $J, H, K$ s channel), the total observing time amounted to about $6 \mathrm{~h}$.

Pixel- and gain-corrected, astrometrized, stacked images for each channel and per $\mathrm{OB}$ were produced using the data reduction and photometry tools of the GROND pipeline (Küpcü Yoldaş et al. 2008; Krühler et al. 2008), based on standard $I R A F^{4} / P y R A F$ tools (Tody 1993). Astrometry was achieved through the use of stars in common with the SDSS catalogue (Abazajian et al. 2009) for the optical bands and the 2MASS catalogue (Skrutskie et al. 2006) for the near-IR ones. For each image the FWHM of the average point-spread-function (PSF) was measured from the surface brightness profiles of randomly distributed non-saturated stars. Images corresponding to values of the average seeing FWHM higher than $1.5^{\prime \prime}$ were not considered in the following analysis. For each band, all selected images were convolved to the worst PSF $\left(\sim 1.5^{\prime \prime}\right.$ FWHM) and then stacked with a median algorithm. The total exposure times of the stacked $g^{\prime}, r^{\prime}, i^{\prime}, z^{\prime}, J, H, K \mathrm{~s}$ images are equal to $11808 \mathrm{~s}$, $14760 \mathrm{~s}, 14760 \mathrm{~s}, 14760 \mathrm{~s}, 12000 \mathrm{~s}, 10800 \mathrm{~s}$, and $9600 \mathrm{~s}$, respectively. The stacked near-IR images were regridded onto the same scale as the optical ones $\left(0.158^{\prime \prime} /\right.$ pixel). All seven stacked

\footnotetext{
${ }^{4}$ IRAF is distributed by the National Optical Astronomy Observatories, which are operated by the Association of Universities for Research in Astronomy, Inc., under cooperative agreement with the National Science Foundation.
}

images were re-mapped to the same aspect with the $z^{\prime}$-image as reference.

Figure 4 reproduces a $J, z^{\prime}, g^{\prime}$ colour-composite (RGB) image of the region of XMMU J0338.7+0030 mapped with GROND (left panel) together with the deep $z^{\prime}$-image (right panel).

\subsubsection{The photometric catalogue of XMMU J0338.7+0030: source extraction and photometry}

A separate stack of seven individual images in the $z^{\prime}$ channel, PSF-matched to the same angular resolution of 1.1" (FWHM), was used as a detection image with a total exposure time equal to $10332 \mathrm{~s}$. This choice was the best compromise between image quality, photometric depth in the red/near-IR channels, robustness of detection and penalty in the source detection and extraction of photometry associated with deblending. It also enabled a better, independent separation between extended and point-like sources along the accessible flux domain.

Automatic source detection and extraction of photometry within an area of $3.9 \times 4.3 \operatorname{arcmin}^{2}$ was accomplished through the publicly available, standard software SExtractor (Bertin \& Arnouts 1996). A source was defined by the excess over a detection threshold equal to the standard deviation $(1 \sigma)$ of the global image background in at least two contiguous pixels; in addition, a Gaussian filter with a FWHM of 1.1" was applied for detection. Photometry of individual sources was extracted in six circular apertures with diameters equal to 1, 2, 3, 4, 5 and 10 arcsec, in a Kron (1980) aperture of factor 2.5 and minimum radius of 3.5 
(SExtractor MAG_AUTO) and in a Petrosian (1976) aperture of factor 2.0 and minimum radius of 3.5 .

In the first step of extraction of source photometry, typical photometric zeropoints for the GROND channels ${ }^{5}$ were assumed together with typical corrections for atmospheric absorption (or airmass), in order to aid identification of stars in common with the existing SDSS and 2MASS catalogues and enable the calibration of the zeropoints of the present $g^{\prime}, r^{\prime}, i^{\prime}, z^{\prime}, J, H, K$ s images. In particular, we made use of the seventh Sloan data release (DR7; Abazajian et al. 2009, and references therein) and the 2MASS All Sky Catalog of point sources (Cutri et al. 2003). After this photometric calibration, adjustments for the newly determined zeropoints were introduced and magnitudes were corrected for Galactic extinction according to the values tabulated in Schlegel et al. (1998) in addition to the previous correction for atmospheric absorption.

For the photometric calibration of the $g^{\prime}, r^{\prime}, i^{\prime}, z^{\prime}$ $(J, H, K s)$ images, the celestial coordinates of GROND sources and SDSS (2MASS) catalogue stars were first cross-correlated using a search radius of 3 ". "Machine" Petrosian magnitudes (corrected only for atmospheric absorption) and catalogue Petrosian magnitudes (not corrected for Galactic extinction) of non-saturated sources that are unambiguously identified as stars and have robust photometry were contrasted after taking into account eventual colour terms (see footnote 5). For a given band, the weighted mean of the magnitude differences was taken as the correction to the assumed zeropoint and the value of the weighted standard deviation as the uncertainty of the photometric zeropoint. These uncertainties are equal to \pm 0.009 , $\pm 0.010, \pm 0.011, \pm 0.011, \pm 0.041, \pm 0.009$ and \pm 0.036 mag in the $g^{\prime}, r^{\prime}, i^{\prime}, z^{\prime}, J, H, K$ s channels of GROND, respectively. They reflect the quality of the reference photometry and the poor statistics of the matched stars used as calibrators. The good agreement between our set of calibrated magnitudes and that of SDSS (2MASS) for all the 38 (9) stars in common is shown in Fig. 5 (6).

Our calibrated Petrosian magnitudes are compared to the SDSS ones (all not corrected for Galactic extinction) for the 65 sources in our photometric catalogue that are associated with galaxies identified in DR7 using a search radius of 3 ". In addition, twenty-four of these 65 SDSS galaxies have a photometric redshift in DR7. The overall agreement between the two sets of Petrosian magnitudes is satisfying (Fig. 5). Unfortunately, no galaxy counterparts to our sources seem to exist in any 2MASS catalogue, according to NED. This is no surprise given the shallow limiting magnitudes of 2MASS (see Jarrett et al. 2001; Skrutskie et al. 2006).

A calibrated photometric catalogue with 832 sources was built as previously described, where magnitudes are corrected for atmospheric extinction and Galactic extinction. Limiting magnitudes ( $1 \sigma$ values) were computed as the median values of magnitudes obtained in $3^{\prime \prime}$ apertures on 10 separated regions of blank sky distributed across the individual $g^{\prime}, r^{\prime}, i^{\prime}, z^{\prime}, J, H, K$ s images. They correspond to 27.30, 26.89, 26.54, 26.06, 24.40, 23.50 and $22.91 \mathrm{mag}$, respectively. We note that the corresponding $5 \sigma$ values in the $z^{\prime}, J, H, K$ s channels are about the depths in analogous bands that the ESO public

\footnotetext{
${ }^{5}$ GROND photometric zeropoints in the $\mathrm{AB}$ magnitude system are available at http://www.mpe.mpg.de/ jcg/GROND/calib.html and in Greiner et al. (2008) together with conversions between different filter systems or from the $\mathrm{AB}$ to the Vega magnitude system. These conversions are equal to $-0.008,-0.151,-0.386$, $-0.515,-0.910,-1.381$ and $-1.795 \mathrm{mag}$ for, respectively, the GROND $g^{\prime}, r^{\prime}, i^{\prime}, z^{\prime}, J, H, K$ s bands.
}
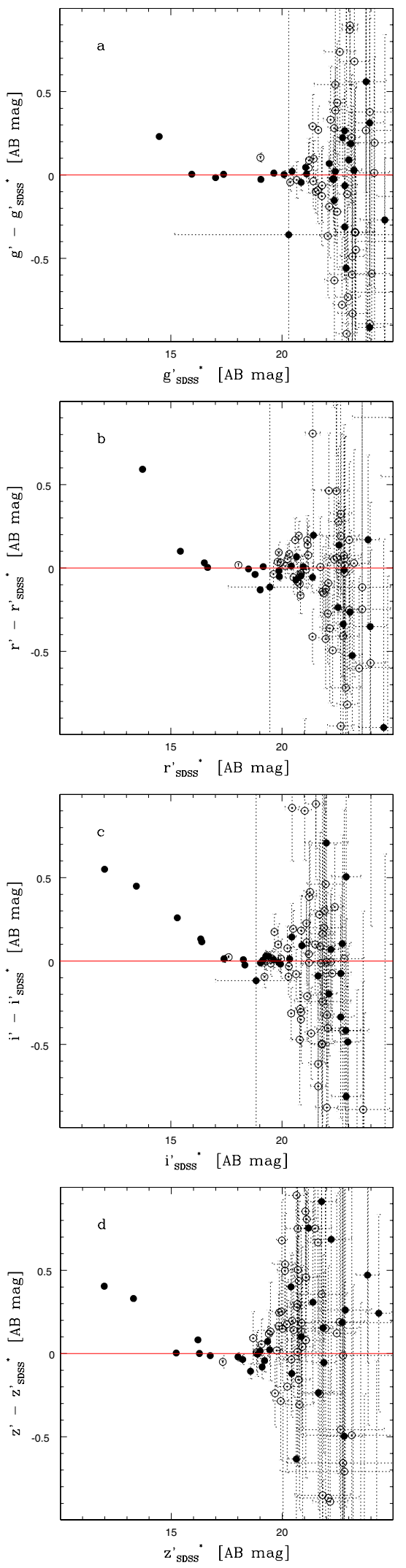

Fig. 5. Comparison of the Petrosian magnitudes of stars (solid circles) and galaxies (empty circles) with GROND and SDSS photometry. The photometry in the GROND $g^{\prime}, r^{\prime}, i^{\prime}, z^{\prime}$ bands is calibrated with that of the stars in common with SDSS in the corresponding SDSS bands (panels a)-d), respectively). Saturated stars and stars with poor photometry were flagged out. The asterisks indicate that the reference magnitudes are expressed in the GROND filter system. In each panel the red solid line represents equality. 

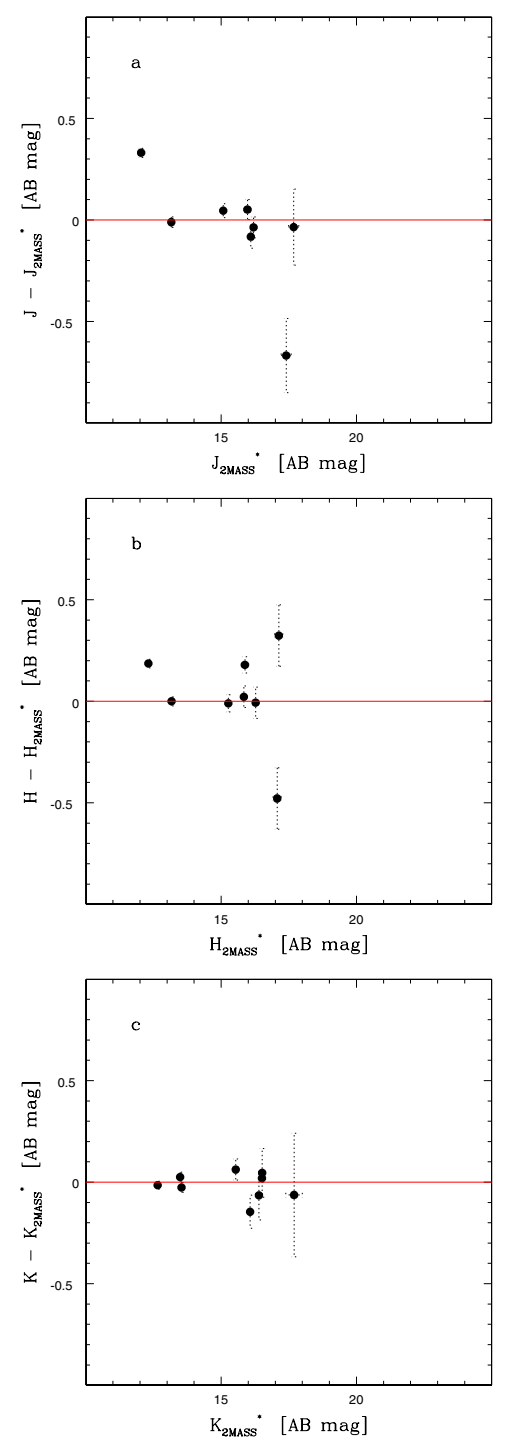

Fig. 6. Comparison of the Petrosian magnitudes of stars (solid circles) after their photometry in the GROND $J, H, K$ s bands is calibrated with that in the corresponding 2MASS bands (panels a)-c), respectively). Saturated stars and stars with poor photometry were flagged out. The asterisks indicate that the reference magnitudes are expressed in the GROND filter system. In each panel the red solid line represents equality.

survey VIKING (VISTA Kilo-Degree Infrared Galaxy Survey; P.I.: W. Sutherland) is expected to reach (see e.g., Arnaboldi et al. 2007).

Corrections to the SExtractor output photometric errors for a given band (in part caused by correlated noise in the stacked images) were computed from the ratio of the median value of the uncertainties of the previous 10 aperture magnitudes and the median SExtractor uncertainty of the $3^{\prime \prime}$ aperture photometry of sources with magnitudes within $\pm 1 \sigma$ from the limiting magnitude. These correction factors amount to $1.74,1.67,1.82$, $1.28,2.15,1.36$, and 1.78 for the $g^{\prime}, r^{\prime}, i^{\prime}, z^{\prime}, J, H, K$ s bands, respectively.

Extended and point-like sources were initially identified on the basis of surface brightness distribution (i.e., the SExtractor parameters stellarity index and flux radius) and apparent brightness. The photometry of point-like sources enabled us to estimate corrections from the $3^{\prime \prime}$ aperture magnitudes to magnitudes in the $z^{\prime}$-band Kron aperture for all bands.
These aperture corrections (in the standard SExtractor format) amount to

$$
\begin{aligned}
& g^{\prime}=g^{\prime}\left(3^{\prime \prime}\right)-0.1143( \pm 0.0094), \\
& r^{\prime}=r^{\prime}\left(3^{\prime \prime}\right)-0.1366( \pm 0.0046), \\
& i^{\prime}=i^{\prime}\left(3^{\prime \prime}\right)-0.1219( \pm 0.0051), \\
& z^{\prime}=z^{\prime}\left(3^{\prime \prime}\right)-0.1254( \pm 0.0040), \\
& J=J\left(3^{\prime \prime}\right)-0.1613( \pm 0.0175), \\
& H=H\left(3^{\prime \prime}\right)-0.1510( \pm 0.0197), \\
& K \mathrm{~s}=K \mathrm{~s}\left(3^{\prime \prime}\right)-0.1463( \pm 0.0307)
\end{aligned}
$$

We have then adopted two operational definitions of total magnitudes and investigated their performances given the characteristics of the available imaging. As a first prescription (method $A$ ), we adopted the Kron magnitude in the $z^{\prime}$ band as the reference total magnitude and computed total magnitudes in the $g^{\prime}, r^{\prime}, i^{\prime}, J, H, K$ s bands from the reference total magnitude plus the corresponding colour term measured within a $3^{\prime \prime}$ aperture and corrected to the Kron aperture. As a second prescription $(\operatorname{method} B)$, we adopted the Kron magnitude as the total magnitude in the $z^{\prime}$ band but computed total magnitudes in the $g^{\prime}, r^{\prime}, i^{\prime}, J, H, K$ s bands from the corresponding $3^{\prime \prime}$ aperture magnitudes, corrected to the Kron aperture. This second set of total magnitudes yielded photometric redshifts that are better consistent with the spectroscopic ones for the three confirmed members of XMMU J0338.7+0030 with GROND photometry (Sect. 3.2.1). At the same time the distribution of these three spectroscopic members in the $i^{\prime}-z^{\prime}$ vs. $z^{\prime}$ colour-magnitude diagram is much better consistent with the red-sequence of the cluster RDCS J0910+5422 at $z=1.106$ (Mei et al. 2006a), as discussed in Sect. 3.2.2. For these reasons, we will make use of the aperture-corrected magnitudes in the $g^{\prime}, r^{\prime}, i^{\prime}, J, H, K \mathrm{~s}$ bands and the Kron magnitude in the $z^{\prime}$ band for all sources (method $\left.B\right)$, and refer to them as total magnitudes hereafter.

Figure 7 illustrates the relation between the computed total magnitude and its uncertainty in each of the GROND $g^{\prime}, r^{\prime}, i^{\prime}, z^{\prime}, J, H, K$ s channels. The regular behaviour observed for the GROND $g^{\prime}, r^{\prime}, i^{\prime}, J, H, K$ s broad bands is due to the fact that two sources hardly exhibit exactly the same measured value for their aperture fluxes. For the GROND $g^{\prime}, r^{\prime}, i^{\prime}, J, H, K$ s channels the uncertainty on the total magnitude is dominated by the uncertanty on the aperture correction at bright magnitudes and by the measurement error at faint magnitudes. The transition between these two regimes corresponds to a total magnitude that depends on the filter.

\subsubsection{The photometric catalogue: star/galaxy separation}

As an alternative to the previous selection criteria, galaxies (extended sources) can be separated from bona fide stars (point-like sources) on the basis of their $g^{\prime}-z^{\prime}$ and $z^{\prime}-K$ s colours, in analogy with the $B z K$ criterion (Daddi et al. 2004; cf. e.g. Galametz et al. 2009). We adopted the formulation of the colour criterion given by Galametz et al. (their Fig. 5) and applied the empirical colour transformation between the SDSS photometry and the Johnson-Cousins photometric system of Jordi et al. (2006)

$g-B=0.370 \times(B-V)-0.124$

plus suitable corrections for the different filter transmission functions and magnitude systems. The ensuing threshold corresponding to the GROND $g^{\prime} z^{\prime} K$ s criterion (short-dashed line in Fig. 8) 
D. Pierini et al.: The galaxy population of XMMU J0338.7+0030 at $z=1.1$
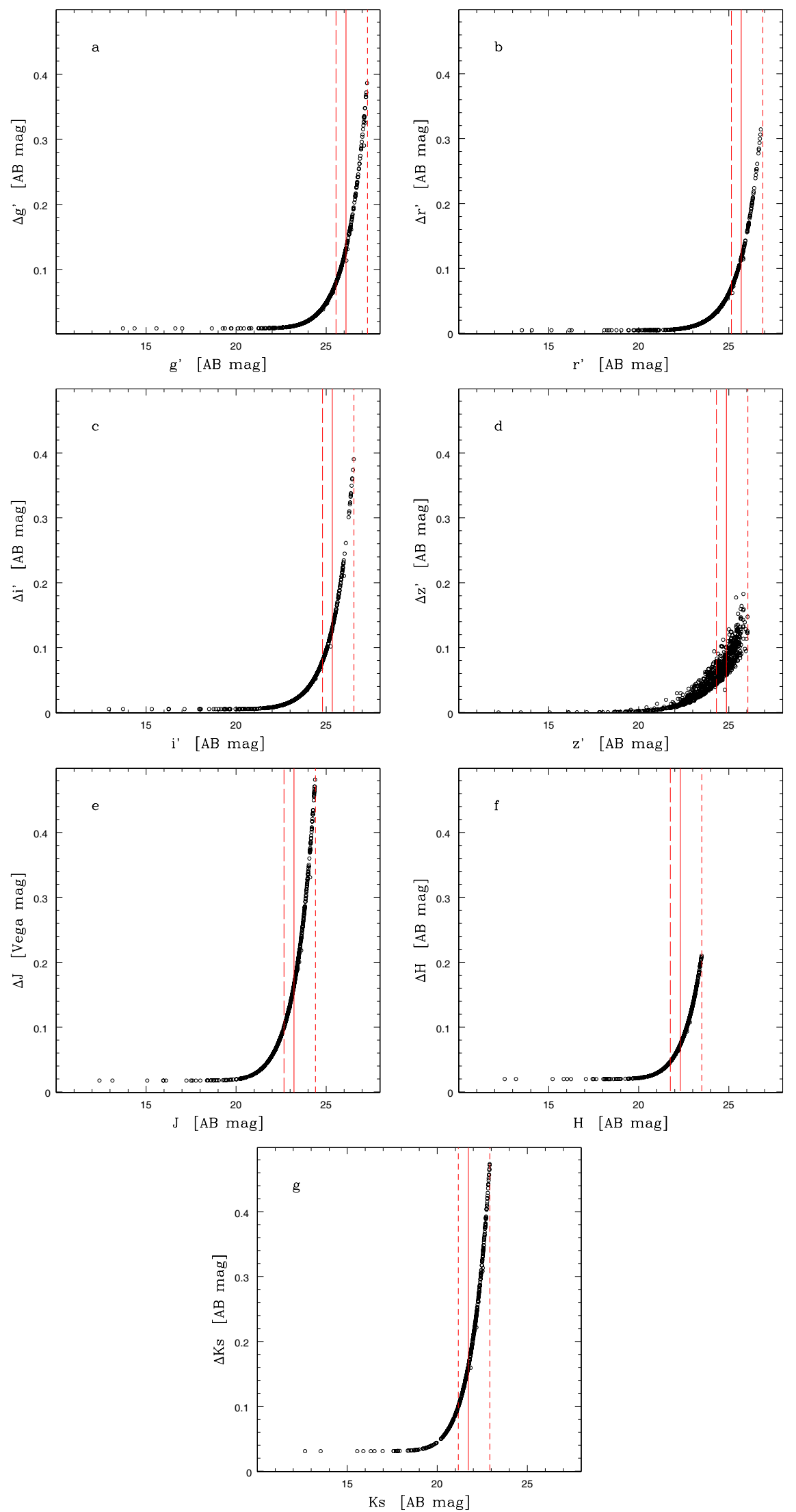

Fig. 7. Uncertainty of the computed total photometry in each of the $g^{\prime}, r^{\prime}, i^{\prime}, z^{\prime}, J, H, K$ s broad-bands of GROND as a function of the total magnitude of a source (panels a)-g), respectively). In each panel the short-dashed, solid and long-dashed red lines represent the $1 \sigma, 3 \sigma$ and $5 \sigma$ limits, respectively. Only sources brighter than the limiting magnitude in a given band are reproduced. 


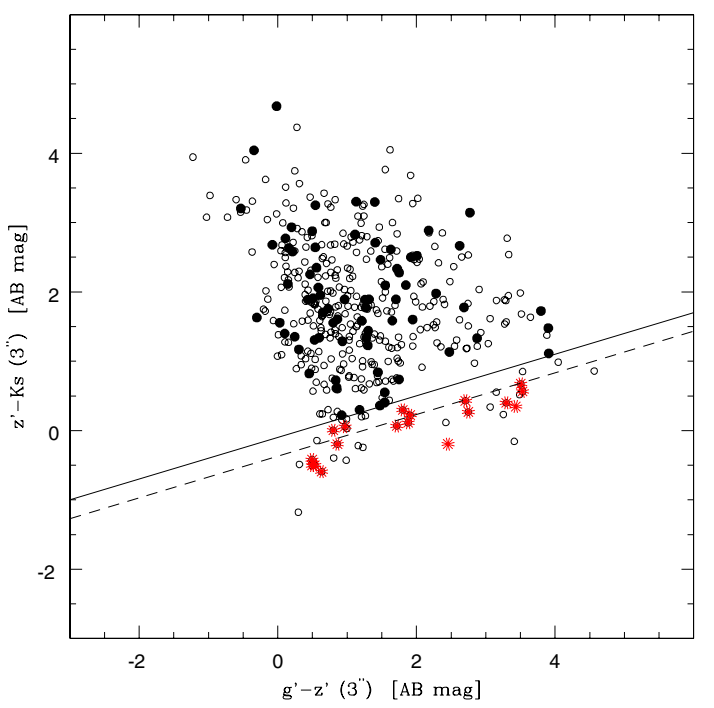

Fig. 8. Distribution of all sources with fluxes in the GROND $g^{\prime}, z^{\prime}, K$ s channels above the respective $1 \sigma$ thresholds in the $z^{\prime}-K$ s vs. $g^{\prime}-z^{\prime}$ colour-colour diagram. Magnitudes within a $3^{\prime \prime}$-wide circular aperture are used. Sources identified as point-like on the basis of a SExtractor stellarity index larger than 0.98 are marked with red asterisks. Conversely, extended sources within (beyond) $1^{\prime}$ from the position of XMMU J0338.7+0030 are marked with filled (empty) circles. The short-dashed and solid lines represent, respectively, the computed and adopted thresholds to select bona fide stars (see text).

was contrasted against the behaviour of our catalogue sources classified as stars on the basis of the SExtractor stellarity index in Sect. 2.3.2. This comparison prompted the introduction of a shift equal to $+0.27 \mathrm{mag}$ in $z^{\prime}-K$ s (solid line in Fig. 8). Therefore, the $g^{\prime} z^{\prime} K$ s criterion hereafter adopted to single out bona fide stars is

$z^{\prime}-K \mathrm{~s}\left(3^{\prime \prime}\right) \leq 0.3 \times\left[g^{\prime}-z^{\prime}\left(3^{\prime \prime}\right)\right]-0.1$,

where we adopted 3 "-aperture measurements. Comparison with the source classification provided by the use of the photometric redshift technique (Fig. 13a in Sect. 3.2.1) reveals the presence of a few extragalactic point-like sources with $g^{\prime}-z^{\prime}\left(3^{\prime \prime}\right) \sim 1$ and $z^{\prime}-K \mathrm{~s}\left(3^{\prime \prime}\right) \sim 0$ that are misclassified as stars in Fig. 8 .

As an independent test of the overall goodness of the previous criterion, in Fig. 9, we compared the distribution of the bona fide stars identified through Eq. (1) in the $J-K$ s vs. $z^{\prime}-K$ s colour-colour diagram with the loci occupied by spectrally classified stars with SDSS and 2MASS photometry (Galametz et al. 2009, their Fig. 1) after introducing suitable corrections for the different filter transmission functions. As discussed by Galametz et al., most of the stars in a matched 2MASS/SDSS catalogue populate either the region of the $J-K$ s vs. $z^{\prime}-K$ s colour-colour diagram above the short-dashed line and tend to be redder than $z^{\prime}-K \mathrm{~s}=0$ if their individual spectral types are later than $\mathrm{K} 5$, or the region below the short-dashed line and across/to the right of the solid line if they are earlier than K5. The consistent behaviour we find confirms the validity of Eq. (1).

\subsection{Optical spectroscopy with FORS2}

\subsubsection{Observations and data reduction}

The SDSS DR7 archive contains no spectroscopy for the region of the sky encompassing XMMU J0338.7+0030. However, sparse spectroscopic information is available from XDCP,

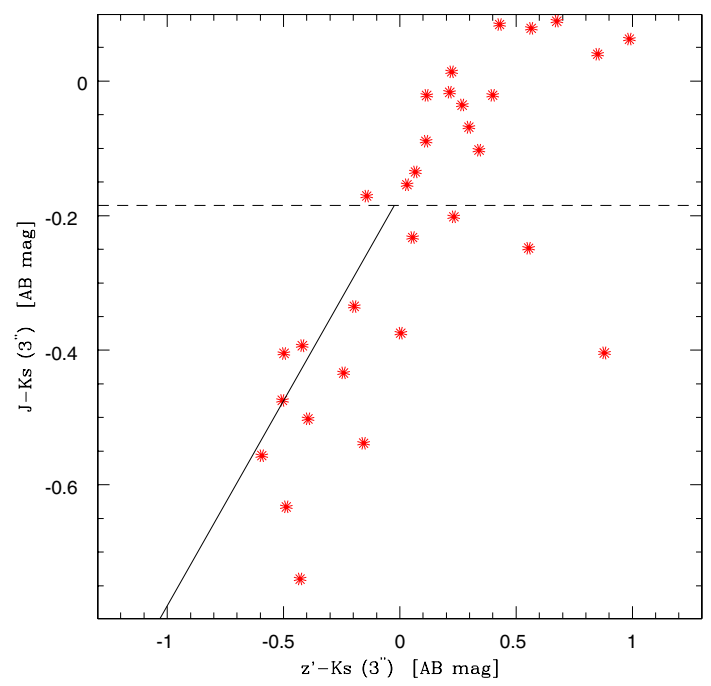

Fig. 9. Distribution of all bona fide stars, selected according to Eq. (1), in the $J-K$ s vs. $z^{\prime}-K$ s colour-colour diagram, where $3^{\prime \prime}$-aperture photometry is used. This distribution is consistent with the loci occupied by spectrally classified stars with SDSS and 2MASS photometry (cf. Fig. 1 in Galametz et al. 2009). As shown by Galametz et al., stars with a spectral type later than K5 populate the region above the short-dashed line and tend to exhibit $z^{\prime}-K \mathrm{~s}>0$. Conversely, stars with a spectral type earlier than K5 populate the region blueward of the horizontal shortdashed line and define a linear colour-colour relation (solid line) with extensions towards very red $z^{\prime}-K$ s colours.

since the neighboring distant cluster XMMU J0338.5+0029 (Fassbender et al. 2011c) was observed on November 7, 2007 (programme ID 079.A-0634, service mode) for $3 \mathrm{~h}$ with FORS2 (Appenzeller et al. 1998). This is a multi-mode (imaging, polarimetry, long slit, MOS) instrument working at wavelengths of 3300-11000 $\AA$, mounted on the Cassegrain focus of VLT UT1 in Paranal, Chile.

A single mask with 32 slits of $1^{\prime \prime}$-width each was designed and used in the FORS2 MXU-mode (Mask eXchange Unit) with the standard resolution collimator, which yields a $6.8^{\prime} \times 6.8^{\prime}$ FoV with a scale of $0.25^{\prime \prime} /$ pixel. Coupled to the 300I grism and the OG590 order blocking filter, this provided a resolution $R=660$ and a wavelength coverage of 6000-10000 A. Six single exposures were taken during the night for the same set-up, yielding a total net integration time of $8400 \mathrm{~s}$; the seeing ranged between $1.2^{\prime \prime}$ and 1.3" (FWHM). Because XMMU J0338.7+0030 was the secondary target of these FORS2 observations, only $30 \%$ of the slits were used to probe galaxies in its region. As primary and secondary spectroscopic targets were chosen, respectively, very red and bluer objects in the $z-H$ vs. $H$ colour-magnitude diagram built out of the OMEGA2000 photometry (Fig. 3). As a result, only five slits out of a total of 32 turned out to correspond to objects in the region of XMMU J0338.7+0030 later imaged with GROND, for which photometry was extracted.

A new version of the VIMOS Interactive Pipeline and Graphical Interface (VIPGI; Scodeggio et al. 2005), modified to make it usable on FORS2 multi-slit spectra, was used. A detailed discussion of this new multi-spectra reduction pipeline is deferred to a forthcoming paper (Nastasi et al., in prep.). In brief, the stacked 2-D raw data were flat-fielded, bias-subtracted and calibrated in wavelength by means of a helium-argon lamp used as a reference line spectrum. The final dispersion solutions had a root-mean-squares calibration uncertainty lower than $1 \AA /$ pixel. The continuum of each spectrum was corrected for the sensitivity function of the FORS2 instrument, and the 1-D reduced 


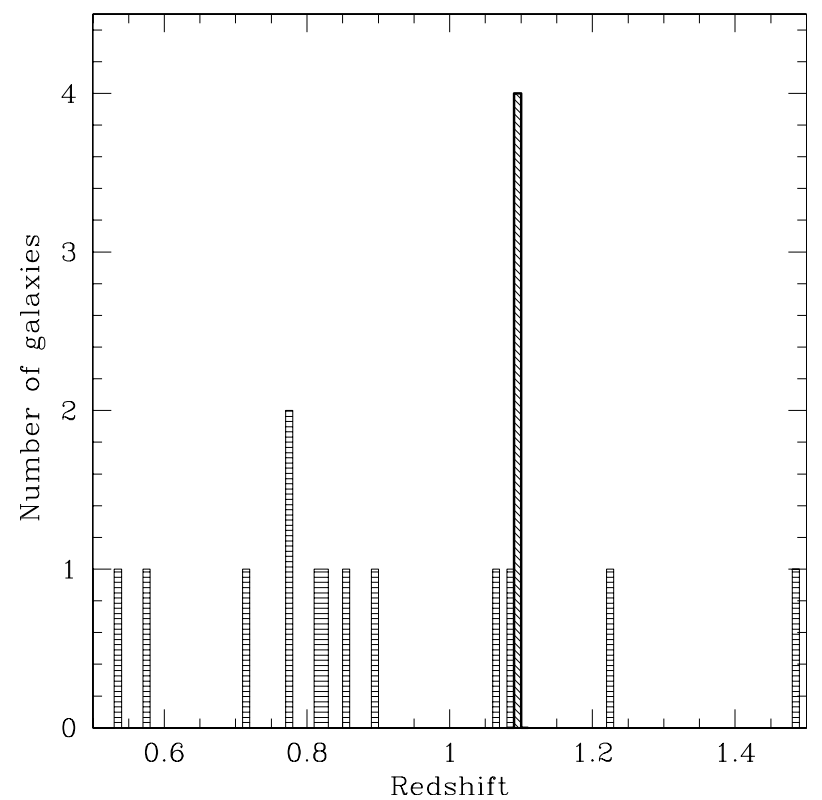

Fig. 10. Histogram of the measured galaxy spectroscopic redshifts in bins of $\Delta z=0.01$. An overdensity at $z=1.1$ is evident. Only five of these redshifts belong to objects in the GROND photometric catalogue.

spectrum was extracted from each slit for the redshift determination. Each 1-D reduced spectrum was both visually inspected by means of the graphical tools available in VIPGI and crosscorrelated with template galaxy spectra using the IRAF package RVSAO (Kurtz \& Mink 1998).

\subsubsection{The spectroscopic redshift of XMMU J0338.7+0030}

Figure 10 shows the redshift distribution of the galaxies at $z \geq 0.5$ that were observed with FORS2 in a $6.8^{\prime} \times 6.8^{\prime}$ region of the sky that contains both XMMU J0338.5+0029 (primary target) and XMMU J0338.7+0030 and that are not members of XMMU J0338.5+0029 (see Fassbender et al. 2011c). Four galaxies exhibit consistent redshifts and are therefore identified as spectroscopic cluster members. As a consequence, XMMU J0338.7+0030 is at a mean spectroscopic redshift $\langle z\rangle=$ $1.097 \pm 0.002(1 \sigma$, where the uncertainty is determined from a boostrap analysis combined with the biweight location estimator. These four spectroscopic members lie within 2.3' from the X-ray position of their host cluster, which corresponds to a projected distance of $\sim 1.1 \mathrm{Mpc}$ for the assumed cosmology (Sect. 1). Their spectra are shown in Fig. 11, where the most prominent spectral features are labelled; their main properties are listed in Table 1.

Out of these four spectroscopic members, three (with ID $=2$, ID $=15$ and ID = 16) have counterparts in the GROND photometric catalogue and lie within $1^{\prime}$ from the original X-ray position of XMMUJ0338.7+0030 (see Fig. 4). The object with ID $=2$ can be identified as a passively evolving galaxy (likely an early-type galaxy) on the basis of its spectral properties. Conversely, the spectroscopic member with ID $=15$ exhibits robust evidence of on-going star-formation activity, as traced by the (unresolved) [O II] doublet at $\lambda=3727 \AA$. This activity is not as vigorous as in the spectroscopic member with $\mathrm{ID}=12$ (modulo dust attenuation) though. The object with ID $=16$ has the least robust determination of photo- $z$ and a controversial evidence of [O II] line emission. It is located in correspondence

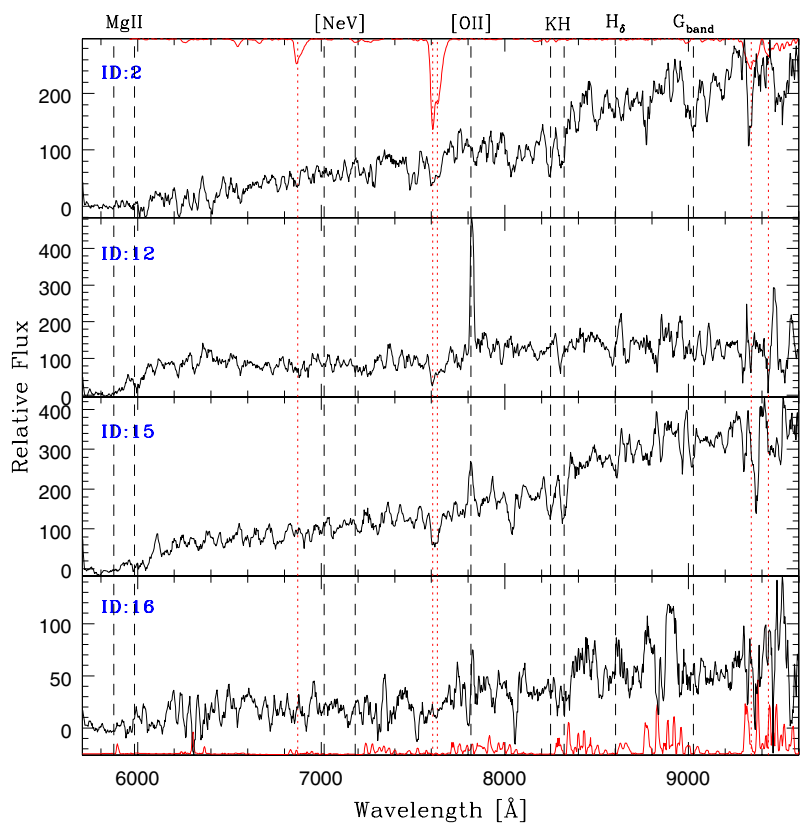

Fig. 11. Spectra of the four cluster members smoothed with a sevenpixel boxcar filter. Black, dashed lines indicate the expected positions of prominent spectral features at $z=1.1$. Features caused by telluric absorption (top panel) or sky emission (bottom panel) are plotted in red.

of the X-ray centroid of XMMU J0338.7+0030 determined in Sect. 2.1.1.

The absence/presence of the [O II] line and its equivalent width $(E W)$ are consistent with the measured values of the strength of the $4000 \AA$ break $(D 4000)$ as shown in Table 1. In particular, a value of $D 4000 \sim 1.9$ is consistent with a minimum light-weighted age of the stellar populations of $\sim 3 \mathrm{Gyr}$, according to the set of models with different star-formation histories and metallicities in Gallazzi et al. (2005) (A. Gallazzi, priv. comm.). Together with the lack of detected [O II] line emission, this confirms the spectroscopic member with ID $=2$ as a galaxy dominated by old, passively evolving stellar populations (i.e., an early-type galaxy). Minimum light-weighted ages of the stellar populations equal to $\sim 1 \mathrm{Gyr}$ and $\sim 1.4 \mathrm{Gyr}$ seem to characterize the spectroscopic members with ID $=15$ and ID $=16$, respectively. These galaxy are likely evolved systems with ongoing star-formation activity. Finally, the spectroscopic member with ID $=12$ exhibits not only the strongest ongoing star-formation activity but also, consistently, the lowest value of $D 4000$, which sets the minimum light-weighted age of its stellar populations to be equal to $\sim 0.3 \mathrm{Gyr}$.

\section{Results}

\subsection{Galaxy number counts}

In order to illustrate depth and completeness of the photometric catalogue extracted from the GROND optical/near-IR imaging (Sect. 2.3.3), the number of galaxies per 0.5 mag-bin and unit area for each of the $i^{\prime}, z^{\prime}, K \mathrm{~s}$ bands is reproduced in Fig. $12^{6}$. Galaxies are selected as in Sect. 2.3.3. Statistical uncertainties are determined from the approximate formulae for confidence

${ }^{6}$ Corrections for masked areas are introduced here; they correspond to $0.8 \%$ of the total area, which is equal to $4.64 \times 10^{-3} \mathrm{deg}^{2}$. Since these corrections are negligible, they will not be introduced later on. 
limits based on the Poisson and binomial statistics presented in Gehrels (1986).

The GROND $i^{\prime}-, z^{\prime}$ - and $K$ s-band galaxy number counts are compared with those determined from observations of deep fields or survey areas like the Hawaii Hubble Deep Field North (Hawaii HDF-N, Suprime-Cam I- and $z^{\prime}$-band - Capak et al. 2004), the Cosmic Evolution Survey (COSMOS, ACS WFC F814W filter - Leauthaud et al. 2007; Suprime-Cam $i^{+}$band - Capak et al. 2007; WIRCam Ks band - McCracken et al. 2010), the Calar Alto Deep Imaging Survey (CADIS, OMEGA Prime $K^{\prime}$ band - Huang et al. 2001), the Subaru Deep Field (SDF, CISCO $K^{\prime}$ band - Maihara et al. 2001 ${ }^{7}$ ), the infrared complement of the VIMOS-VLT deep survey (VIRMOS, SOFI $K$ s band - Iovino et al. 2005), the FLAMINGOS Extragalactic Survey (FLAMEX, FLAMINGOS Ks band - Elston et al. 2006), and the UKIRT Infrared Deep Sky Survey Ultra-deep survey (UKIDSS UDS, UKIRT Ks band - Hartley et al. 2008).

The present GROND $z^{\prime}$-selected sample of galaxies has a $50 \%$ completeness limit equal to $z^{\prime \text { compl }} \sim 25.3$, as determined from the extrapolation of the galaxy number counts in Capak et al. (2004). This completeness magnitude corresponds to a $\sim 2 \sigma$ flux detection (cf. Fig. $7 d$ ). The $50 \%$ completeness limit is equal to $\sim 24.8 \mathrm{AB}$ mag in $i^{\prime}$-band, which corresponds to a $\sim 5 \sigma$ flux detection. Finally, the GROND photometric sample is $50 \%$ complete down to $K \mathrm{~s}^{\mathrm{compl}} \sim 22.9$, which corresponds to a $\sim 1 \sigma$ flux detection. This completeness magnitude also corresponds to $\sim K \mathrm{~s}^{\star}+2.4$, where $K \mathrm{~s}^{\star}=20.5_{-1}^{+0.4}$ is the characteristic magnitude (Schechter 1976) of the Ks-band luminosity function of cluster galaxies at $z \sim 1.2$ (Strazzullo et al. 2006).

Comparison of the different galaxy number counts in Fig. 12 shows an overall agreement between our results and those in the literature down to the completeness limit of the GROND photometric sample of galaxies in each band. This is important when considering the differences in the sky area that is probed by different observations (leading to cosmic variance), the operational definition of total magnitude and the adopted galaxy/star separation criteria. These differences mostly affect the bright end of the galaxy number counts, which corresponds to magnitudes approximately brighter than $20 \mathrm{AB}$ mag in Fig. 12. Furthermore, our galaxy number counts refer to the same sample extracted in the $z^{\prime}$-band, whereas the galaxy number counts in the literature refer to individual samples extracted in the $i^{\prime}-, z^{\prime}$ - or $K$-band.

In spite of the overall agreement, our number counts exhibit an excess (by a factor of almost two) in the magnitude range between $K \mathrm{~s}^{\text {compl }}-2$ and $K \mathrm{~s}^{\text {compl }}-0.5$ (i.e., $20.8 \leq K \mathrm{~s} \leq 22.3$ ) with respect to all others. This magnitude range corresponds to $\sim K \mathrm{~s}^{\star}+0.3 \leq K \mathrm{~s} \leq K \mathrm{~s}^{\star}+1.8$ for cluster galaxies at $z \sim 1.2$ (Strazzullo et al. 2006). Consistently, a slight excess across a broader magnitude range (i.e., approximately at magnitudes between 21.5 and $23.5 \mathrm{AB}$ mag) is visible from comparison of the galaxy number counts for the $z^{\prime}$ band. No clear excess is apparent in the galaxy number counts for the $i^{\prime}$-band. This behaviour is interpreted as an indication for the presence of an over-density of galaxies associated with the weak, marginally extended X-ray emission corresponding to XMMU J0338.7+0030.

\subsection{Photometric-redshifts}

The photometric redshift technique enables us to assess the membership to XMMUJ0338.7+0030 for the 832 sources in the GROND photometric sample. Distances to individual

\footnotetext{
7 We plot the completeness-corrected, $K^{\prime}$-band galaxy number counts provided by Maihara et al. (2001).
}
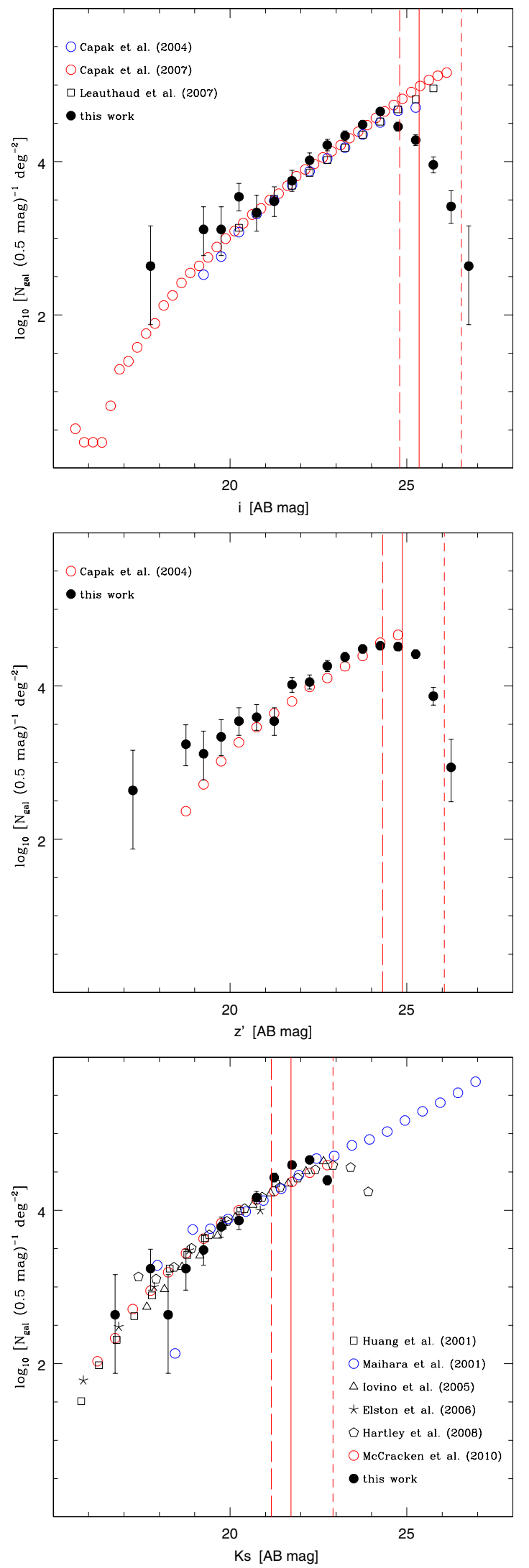

Fig. 12. Comparison of the galaxy number counts determined in the region of XMMU J0338.7+0030 imaged with GROND for the $i^{\prime}$ (top), $z^{\prime}$ (centre) and Ks band (bottom) and the corresponding ones from deep fields or survey areas. Error bars are shown only for our points. In each panel, the short-dashed, solid and long-dashed lines represent the $1 \sigma$, $3 \sigma$ and $5 \sigma$ thresholds of our photometry, respectively. The GROND galaxy number counts exhibit a clear excess for $20.8 \leq K$ s $\leq 22.3$. 
sources were estimated through the publicly available package for PHotometric Analysis for Redshift Estimations, le Phare ${ }^{8}$ (Arnouts \& Ilbert), which is a set of Fortran programmes to compute photo-z's using the standard SED fitting technique.

We selected the large pool of observed SEDs of stars (254) and the mixture of observed and synthetic SEDs for QSOs (28) that are provided by le Phare, as it is standard. However, we initially adopted two sets of templates to describe observed SEDs of galaxies and investigated their performances.

The first set was used for the COSMOS photo- $z$ paper (Ilbert et al. 2009). It contains 31 templates: the SEDs numbered from 1 to 7 correspond to galaxies that can be morphologically classified as elliptical (E), the SED number 8 corresponds to a lenticular (or S0) galaxy, SEDs numbered from 9 to 12 correspond to bulge-dominated disc galaxies (i.e., $\mathrm{Sa}$ and $\mathrm{Sb}$ ), SEDs numbered from 13 to 19 correspond to bulge-less disc galaxies (i.e., $\mathrm{Sc}, \mathrm{Sd}$, and Sdm), SEDs numbered from 20 to 31 describe starburst (SB) galaxies. We followed the indicated prescriptions for the model-dependent attenuation ${ }^{9}$ of galaxy templates, so that a "Prevot extinction law" (Prevot et al. 1984) was applied to SEDs numbered from 13 to 23, a so-called "Calzetti attenuation law" (Calzetti et al. 2000) and two "modified" Calzetti attenuation laws (see Ilbert et al. 2009) were applied to SEDs numbered from 23 to 31 . This choice accounts for the fact that high- $z$ starforming galaxies are characterized by extinction laws that differ at rest-frame ultraviolet wavelengths, as it is in the Local Group (Noll et al. 2009 and references therein). In all cases, the reddening $E(B-V)$ was enabled to range between 0 and 0.5 mag in steps of 0.05 mag.

The second set of galaxy templates corresponds to the 66 SEDs that are used in Ilbert et al. (2009) for the analysis of the CFHTLS Deep fields. In the CFHTLS set, the ratio between the numbers of $\mathrm{E}$ and SB templates is reversed with respect to the COSMOS set: E, Sbc, Scd, Im and SB galaxies are described by 22, 17, 12, 11, and 4 SEDs, respectively. For Scd, Im and SB galaxy templates, a Prevot et al. (1984) extinction law is adopted, with values of $E(B-V)$ ranging from 0 to 0.25 with a step of 0.05 consistently with the indications in the le Phare documentation.

For each library of galaxy SEDs, we considered two separate families of models, by including the contribution to the total flux from line emission or not. We opted to do this because there is independent evidence that the bright end of the galaxy luminosity function of a high- $z$ cluster can be populated by [O II] line emitters (as for XMMU J0302.2-0001 at $z=1.186$, Suhada et al. 2011), sometimes with vigorous starburst activity (as for XMMU J1007.4+1237 at $z=1.555$, Fassbender et al. 2011b).

Finally, for galaxy templates as well as for QSO templates, we considered the redshift range $0-6$ with a step of 0.02 in $z$ and the range $-24 \leq M_{z^{\prime}} \leq-8$ in the $z^{\prime}$-band absolute magnitude.

For all stellar, QSO and galaxy templates, synthetic photometry was computed for all GROND bands in the AB magnitude system and confronted with the observed photometry. Both systematic (e.g., caused by calibration uncertainties) and statistical

\footnotetext{
8 wWw . oamp.fr/people/arnouts/LE_PHARE.html

9 We note that the extinction curve describes the combined absorption and out-of-the beam scattering properties of a mixture of dust grains of given size distribution and chemical composition in a screen geometry as a function of wavelength; the attenuation function is the combination of the extinction curve with the geometry of a dusty stellar system, in which a substantial fraction of the scattered light is returned to the line of sight (e.g. Pierini et al. 2004b).
}

(i.e., caused by measurement uncertainties) errors were taken into account, the latter being magnified by $50 \%$ (in mag).

The photometric redshift code le Phare, based on a simple $\chi^{2}$ fitting method, yields the best-fitting template in each class, the best-fit value of $z$, the $68 \%$ range of the photo- $z$ solutions and the probability per redshift bin, in particular. All ouputs for individual detected sources were inspected by eye. A source was classified as a star if the best-fitting stellar template gave the lowest $\chi^{2}$ value. Conversely, it was classified as a QSO if the bestfitting QSO template gave a $\chi^{2}$ value that was better by a factor of two than those given by the best-fitting stellar and galaxy templates.

As a first step, we checked the reliability of the publicly available transmission functions of the GROND dichroic filters uploaded into le Phare by comparing the distributions of the library stars and the stars observed by us with the most accurate photometry in a large suite of colour-colour diagrams (see Fig. 13 for examples). Eventual problems affecting the GROND photometry of each star in individual bands (e.g., owing to poor $\mathrm{S} / \mathrm{N}$ and deblending) were inspected: if there were compromised photometric data, they were excluded. As a result, we found an overall good agreement between observed and synthetic stellar tracks, which required the addition of very small shifts $(-0.05 \mathrm{mag},+0.025 \mathrm{mag},+0.025 \mathrm{mag})$ to the synthetic photometry in the $r^{\prime}, i^{\prime}, H$ bands, respectively. Furthermore, the colour criterion adopted to statistically select stars from $g^{\prime}, z^{\prime}, K$ s photometry only (Eq. (1)) was nicely confirmed (cf. Figs. 8 and 13a).

As a second step, we computed photometric redshifts for two photometric catalogues with the same sources as part of the test to determine the best operational definition of total magnitude for our data. This test was executed with the COSMOS set of galaxy templates excluding the line emission contribution; in particular, it addressed the five galaxies with GROND photometry and a spectroscopic redshift (Sect. 2.4.1). Overall the uncertainty on the ensuing two sets of photometric redshifts obtained for these five galaxies is similar, as well as the best-fit solutions yielded for the two foreground galaxies. However, a mean photometric redshift equal to $\sim 1.22 \pm 0.25(1 \sigma)$ or $\sim 1.02 \pm 0.15(1 \sigma)$ is obtained for XMMU J0338.7+0030 when total magnitudes were computed using the operational definitions $A$ and $B$ in Sect. 2.3.2, respectively. Because this cluster is spectroscopically confirmed to be at $z=1.1$ (Sect. 2.4.2), the operational definition of total magnitude $B$ has to be favoured. This choice is also supported by the distribution of the three spectroscopic members in the $i^{\prime}-z^{\prime}$ vs. $z^{\prime}$ colour-magnitude diagram (Fig. 15). Therefore, we only discuss results from the fiducial photometric catalogue where total magnitudes are computed with method $B$ hereafter.

Finally, we compared the performances of the COSMOS and CFHTLS sets, whether the emission line contribution to the templates of star-forming galaxies is included or not. We based our judgement on the comparison of the best-fit solutions and their uncertainties but also on the capability of the set of choice to recover the redshifts of the five galaxies with available spectroscopy (see Sect. 2.4.1). This figure corresponds to only $\sim 0.6 \%$ of the GROND photometric sample of 832 sources; the ensuing span in redshift is very narrow: $z=$ 0.85-1.1. Nevertheless, the results obtained for the three spectroscopic members of XMMU J0338.7+0030 (see Sect. 2.4.2) gave us useful indications. The use of the COSMOS galaxy templates yielded a mean photometric redshift equal to $\sim 1.02 \pm$ 0.15 for this cluster, whether emission lines were included or not. Conversely, the use of the CFHTLS galaxy templates yielded values of $\sim 1.14 \pm 0.11$ and $\sim 1.12 \pm 0.09$, respectively. 

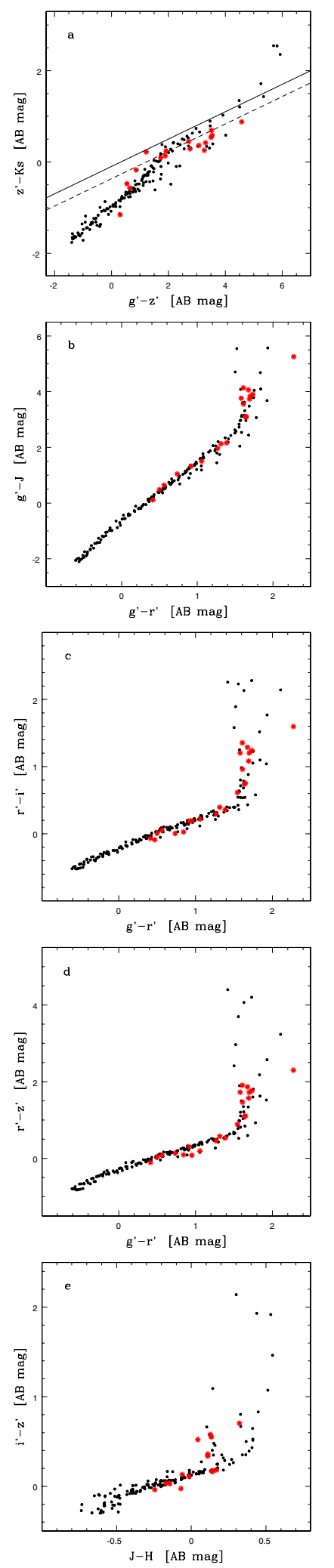

Fig. 13. Five colour-colour diagrams for stars in the le Phare library (black filled circles) and the 15 to 23 objects detected at all GROND bands, with the most accurate photometry in the relevant bands and robustly classified as stars by le Phare using the full seven-channel photometry (red asterisks). These observed stars nicely fall onto the synthetic stellar sequences, after the synthetic photometry is slighlty corrected by $-0.05,+0.025$ and $+0.025 \mathrm{mag}$ in the $r^{\prime}, i^{\prime}$ and $H$ bands, respectively. We recall that photometric redshifts are particularly sensitive to the $4000 \AA$ break in the spectrum of a galaxy, which spans the entire wavelength range of the $z^{\prime}$ broad-band filter for $z=1.1-1.6$.
Given the better agreement with the spectroscopic redshift of XMMU J0338.7+0030 $(\langle z\rangle=1.097 \pm 0.002)$ and the lower uncertainty, hereafter we discuss results obtained from the use of the CFHTLS set of templates without emission line contribution $^{10}$.

As a consequence of this choice, out of the 832 entries in the GROND photometric catalogue, 174 sources do not have a (reliable) photo- $z$ estimate (owing to very poor photometry or multiple peaks in the photo- $z$ probability distribution function), 64 sources are identified as stars, 158 sources exhibit SEDs that are best-fit by a QSO template, 436 sources are identified as galaxies. On one hand, the past large percentage of objects identified as QSOs reflects our arbitrary classification criterion of the stellar, galaxy and QSO templates that best-fit the SED of a given object. On the other hand, it reflects the reduced capability of discriminating between best-fit templates for the numerous objects with incomplete photometric information. For the majority of the objects identified as QSOs, the photometric redshift is reassuringly consistent, if not similar, to the value associated with the best-fit galaxy template.

The quality of the best-fit photometric redshifts obtained with CFHTLS galaxy templates not including emission lines is discussed in Appendix A. The distribution of these photo- $z$ solutions is often single peaked and narrow when accurate photometry is available for all seven GROND channels.

\subsection{Optical identification of the high-z cluster}

\subsubsection{The photometric-redshift distribution function}

We computed the surface density of extragalactic sources within or beyond a cluster-centric distance of $1^{\prime}$, as well as across the entire $3.9 \times 4.3 \mathrm{arcmin}^{2}$ region of the sky imaged with GROND, as a function of the photometric redshift (Fig. 14a). A simple visual inspection reveals that for the redshift bins 1.0-1.1, 1.2-1.3 and $1.5-1.6$, the density of sources within the bona fide cluster region exceeds that computed in the adjacent field by a factor of 2 . An equivalent over-density of $i^{\prime}-K$ s-selected extremely red objects (EROs) ${ }^{11}$ exists in each of the last two bins (Fig. 14b). These EROs are found in the redshift range where they are expected to be, which gives additional confidence in the quality of our photo-z's, at least in a statistical sense.

In support of this conclusion, the spectroscopic members with $\mathrm{ID}=2, \mathrm{ID}=15$ and $\mathrm{ID}=16$ have photometric redshifts ${ }^{12}$ equal to $1.0110_{0.9918}^{1.0311}, 1.1711_{1.1490}^{1.1939}$ and $1.1820_{1.1378}^{1.2461}$, respectively. Furthermore, the morphological type of the best-fitting template corresponds to an E galaxy for the spectroscopic members with $\mathrm{ID}=2$ and ID $=16$ and to an Scd galaxy for the spectroscopic member with ID $=15$. This spectro-photometrically determined classification is nicely consistent with the spectroscopic classification in Sect. 2.4.2. Furthermore, it is reasonable to conclude

10 This suggests that the COSMOS galaxy templates are less adequate to represent the characteristics of the galaxy populations of XMMU J0338.7+0030. An analogous point was made by Guennou et al. (2010), who discussed photometric redshifts along lines of sight to 10 clusters at spectroscopic redshifts between 0.48 and 0.79 obtained with the same COSMOS galaxy templates and photo- $z$ code used here.

11 Colours redder than $I-K=4$ Vega mag statistically select EROs at $z \sim 1-2$ (Pozzetti \& Mannucci 2000, and references therein; see also Pierini et al. 2004a, 2005). For the GROND channels, this corresponds to $i^{\prime}-K \mathrm{~s}=2.8$ in the $\mathrm{AB}$ magnitude system.

12 Here we give the best-fit photo- $z$ together with the values that bracket the $68 \%$ range of the photo- $z$ solutions. 

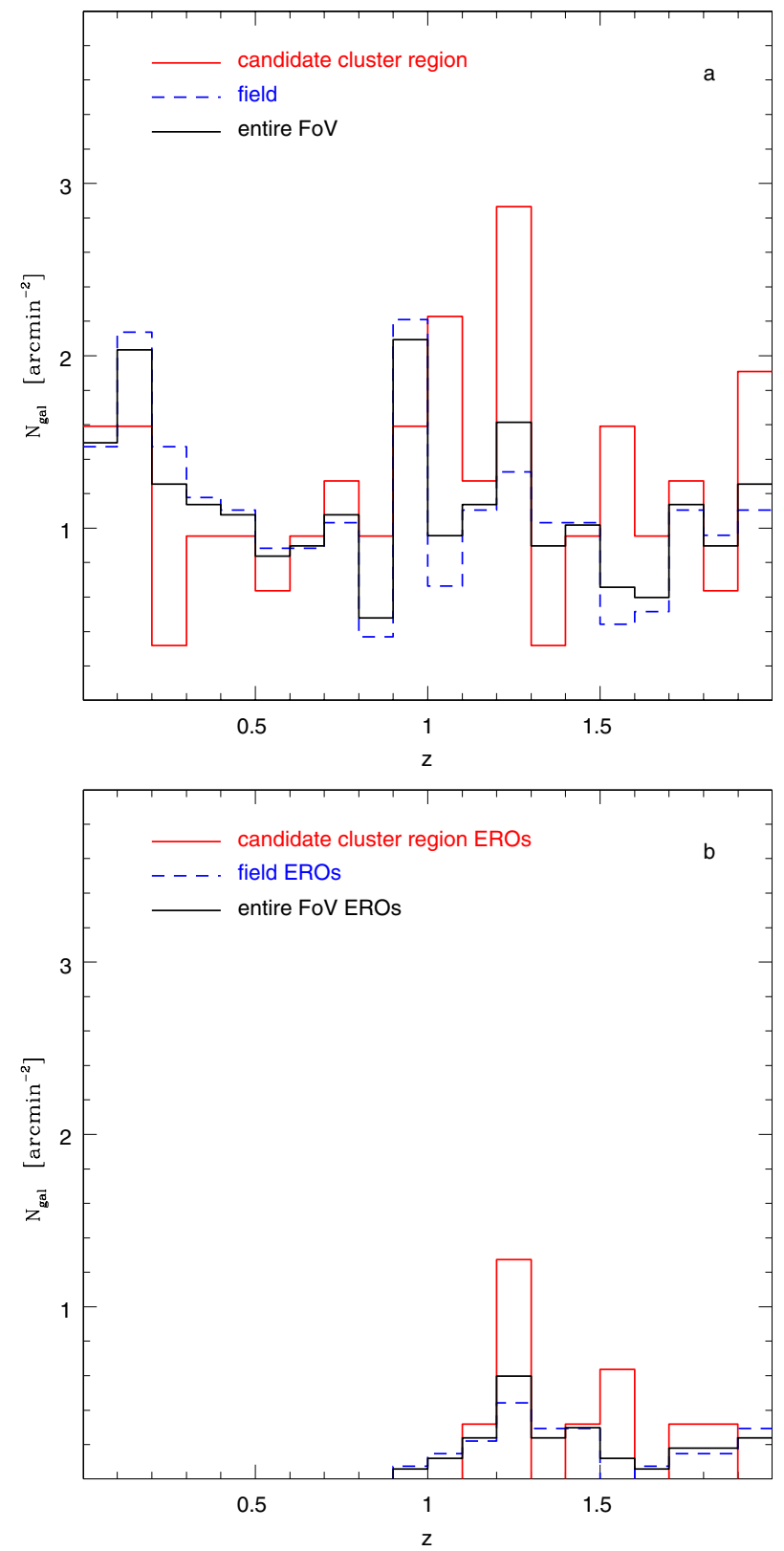

Fig. 14. Number density as a function of the photometric redshift for the extragalactic sources with a photo- $z$ less than 2 in the entire $3.9 \times 4.3 \operatorname{arcmin}^{2}$ region of the sky imaged with GROND (black solid line), for those among them inside the bona fide cluster area (red solid line) or in the surrounding field (blue short-dashed line) - panel a). The analogous distribution functions for the sub-sample of EROs, expected to be at $z \sim 1-2$ (see text), are also reproduced (panel b)). For the redshift bins $1.0-1.1,1.2-1.3$ and $1.5-1.6$, the density of sources within a cluster-centric distance of $1^{\prime}$ exceeds that computed in the adjacent field by a factor of 2 . In correspondence of the last two redshift bins, there is a clear excess of EROs.

that the two peaks at $z \sim 1.0-1.1$ and $z \sim 1.2-1.3$ in Fig. 14a are caused by true cluster members, at least in part.

Therefore we selected as photometric (also candidate or fiducial hereafter) cluster members those sources with a photo- $z$ in the range 1.0110-1.2317, which is centred on the mean photometric redshift of XMMU J0338.7+0030 $(\langle z\rangle=1.1214 \pm 0.0957$, $1 \sigma)$, contains the previous three spectroscopic members and, thus, has a width equal to $\pm 1.15 \sigma$ (or $\pm 0.1\langle z\rangle$ ). Forty-four sources satisfy our criterion: fourteen (36) are within $1^{\prime}\left(2^{\prime}\right)$ from the X-ray position of XMMU J0338.7+0030. Only two of these 44 candidate cluster members are identified as QSOs according to their best-fit templates; they are at cluster-centric distances greater than $1^{\prime}$. The remaining 42 fiducial cluster members are spectro-photometrically classified as E (7), Sbc (6), Scd (11), Im (8) or SB (10) according to the templates that best fit their individual SEDs and provide their fiducial photometric redshifts. As discussed in Appendix A, the photometric redshifts associated with these 42 galaxies are, in general, well-behaved and exhibit low uncertainties.

\subsubsection{The $i^{\prime}-z^{\prime}$ vs. $z^{\prime}$ colour-magnitude diagram: evidence for a red sequence}

We now investigate if the previously selected candidate cluster members consistently define a red sequence in a suitable colour-magnitude diagram. We considered the $i^{\prime}, z^{\prime}$ bands because the rest-frame $4000 \AA$ break spans the entire wavelength range of the $z^{\prime}$ band as the redshift of a source increases from 1.1 to 1.6 . Figure 15 shows the distribution of all sources of the GROND photometric sample in the $i^{\prime}-z^{\prime}$ vs. $z^{\prime}$ colour-magnitude diagram. In the upper panel, the photometric members of XMMU J0338.7+0030 are marked in red or green if they fall inside or outside the bona fide cluster region, respectively. In the lower panel, the same sources are colour-coded according to the best-fit template (if they are identified as galaxies). About a dozen candidate cluster members identified as galaxies (including the three spectroscopic cluster members) suggest the existence of a red locus in the $i^{\prime}-z^{\prime}$ vs. $z^{\prime}$ colour-magnitude diagram: $i^{\prime}-z^{\prime}$ slightly decreases from $\sim 1.1 \mathrm{AB}$ mag to $\sim 1.0 \mathrm{AB}$ mag as $z^{\prime}$ increases from $\sim 21.5 \mathrm{AB}$ mag to $\sim 24.0 \mathrm{AB}$ mag. Furthermore, half of these galaxies are identified as ellipticals. This is consistent with the existence of a red sequence (Arimoto \& Yoshii 1987).

As a reference, the red sequence of RDCS J0910+5422 at $z=1.106$ (Mei et al. 2006a) is reproduced in both panels of Fig. 15. Mei et al. obtained high-resolution imaging of this massive cluster with the Hubble Space Telescope Advanced Camera for Surveys (HST ACS) in the F775W and F850LP bandpasses. They computed total $z_{850}$ magnitudes (SExtractor MAG_AUTO) and $i_{775}-z_{850}$ colours within the effective radii of the individual galaxies in order to select candidate cluster members. The use of these aperture colours was meant to avoid a selection bias introduced by the potential presence of radial colour gradients. Furthermore, Mei et al. fitted different $i_{775}-z_{850}$ vs. $z_{850}$ colour-magnitude relations to different subsamples of morphologically classified early-type galaxies. We have transformed their best-fit relation for the $31 \mathrm{E}+\mathrm{S} 0+\mathrm{S} 0 / \mathrm{a}$ galaxies within 1' from the X-ray centroid of RDCS J0910+5422 (see Table 1 in Mei et al. 2006a) into a red sequence in the $i^{\prime}-z^{\prime}$ vs. $z^{\prime}$ colour-magnitude diagram by introducing corrections for the different transmission functions of the corresponding HST ACS and GROND filters. In spite of the uncertainties on these colour transformations (likely of the order of $0.1 \mathrm{mag}$ ), the red-sequence of RDCS J0910+5422 describes the red locus of XMMU J0338.7+0030 rather well.

However, galaxies with very different spectro-photometric types populate the red locus of XMMU J0338.7+0030 within a cluster-centric distance of $1^{\prime}$ and not just early-type galaxies as in RDCS J0910+5422. This was also observed in e.g. XMMUJ1230.3+1339 at $z=0.975$ (see Fig. 7 in Lerchster et al. 2010). Nevertheless the two coeval clusters XMMU J0338.7+0030 and RDCS J0910+5422 seem to host 

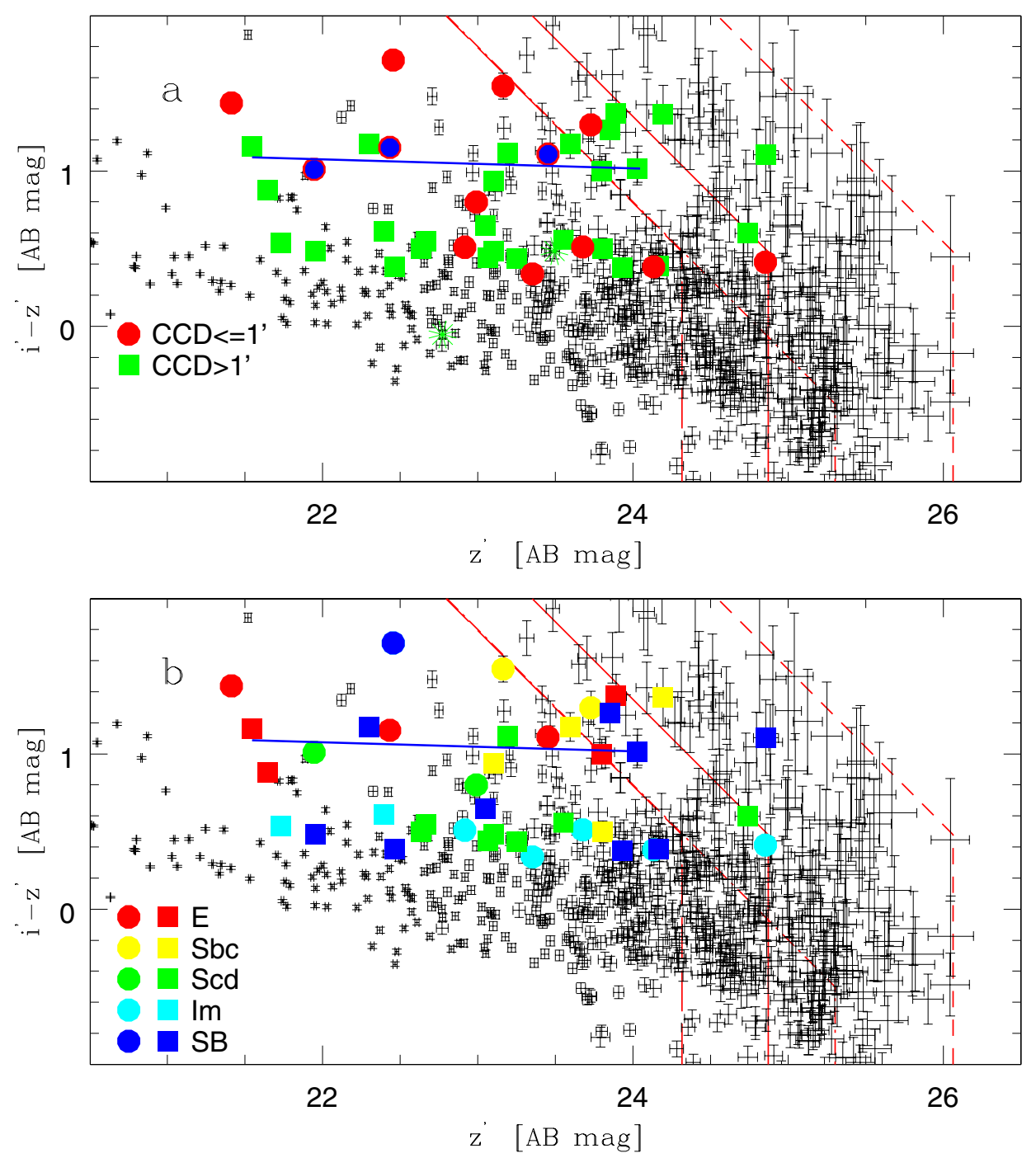

Fig. 15. $i^{\prime}-z^{\prime}$ vs. $z^{\prime}$ colour-magnitude diagram for all GROND sources in the XMMU J0338.7+0030 region with flux detections in the $i^{\prime}, z^{\prime}$ bands at a significance level $\geq 1 \sigma$, whatever the classification of the source by Le Phare (i.e., stars are included). Photometric errors ( $1 \sigma)$ are also shown. Candidate members of XMMU J0338.7+0030 are reproduced with red (green) filled circles (squares) if they are within (outside) the bona fide cluster region (panel a)). The three spectroscopic cluster members in the GROND photometric catalogue are marked with blue filled circles there. Green asterisks represent the two QSO at a cluster-centric distance (CCD) greater than 1'. Alternatively (panel b)), photometric cluster members classified as galaxies are colour-coded according to their spectro-photometric type, which is E (red), Sbc(yellow), Scd (green), Im (cyan) or SB (blue). In each panel, the red short-dashed, solid and long-dashed lines represent the $1 \sigma, 3 \sigma$, and $5 \sigma$ flux thresholds of our photometry (cf. Sect. 2.3.2). The red dotted-short-dashed line represents the completeness limits in the $i^{\prime}, z^{\prime}$ bands, as obtained from Fig. 12. As a reference, the blue solid line reproduces the red-sequence defined by the inner regions of the $31 \mathrm{E}+\mathrm{S} 0+\mathrm{S} 0 /$ a galaxies that are located within $1^{\prime}$ from the $\mathrm{X}$-ray centroid of the cluster RDCS J0910+5422 at $z=1.106$ (Mei et al. 2006a).

also analogus populations of star-forming galaxies that exhibits bluer $i^{\prime}-z^{\prime}$ colours with respect to the red, passively evolving members by about $0.5 \mathrm{AB}$ mag (cf. Fig. 7 in Mei et al. 2006a). For XMMU J0338.7+0030, these galaxies constitute about half of the candidate members and are identified as Scd, Im or SB. Furthermore, those within the bona fide cluster region span the magnitude range $22.9 \leq z^{\prime} \leq 24.9$, whereas those farther out can be as bright as $z^{\prime} \sim 21.7$. These bright, starforming, candidate cluster-member galaxies might be analogous to the spectroscopic member with ID $=12$ at a cluster-distance of 2.3' (cf. Table 1). Evidence of a population of bright starforming disc galaxies was found in RDCS J0910+5422 as well and within a cluster-centric distance of $2^{\prime}$ (Mei et al. 2006a).

In conclusion, the photometric redshifts determined in Sect. 3.2 confirm that XMMUJ0338.7+0030 is a cluster at $z=1.1$ and suggest that its galaxy population is qualitatively similar to that of the coeval cluster RDCS J0910+5422.

\subsubsection{Projected galaxy distribution}

In the entire region of XMMUJ0338.7+0030 imaged with GROND at all seven bands, there are 44 candidate cluster members, including three spectroscopic members (Sect. 3.2.1). Their projected distribution as a function of cluster-centric distance (up to $2^{\prime}$ ) is reproduced in Fig. 16. There are 14 photometric members in the bona fide cluster region and 22 in the concentric annulus of inner and outer radii equal to $1^{\prime}$ and $2^{\prime}$ that has a three times larger area. If one assumes this annulus to represent the surrounding low-density region (i.e., the "field"), the over-density of galaxies within $1^{\prime}$ from the X-ray position 


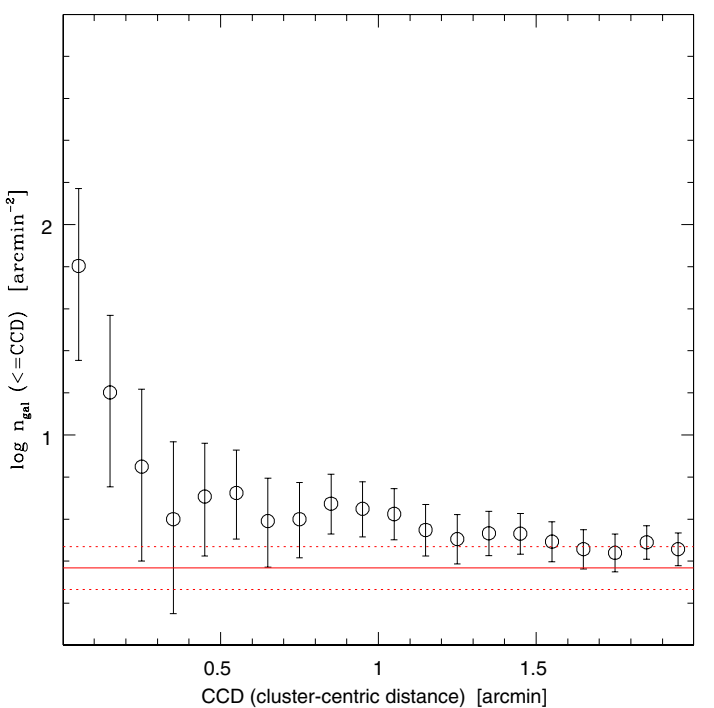

Fig. 16. Cumulative number density of the 44 photometric members of XMMU J0338.7+0030 as a function of their cluster-centric distances. The mean number density of those members within a concentric, circular annulus delimited by radii of $1^{\prime}$ and $2^{\prime}$ is reproduced together with upper and lower limits (red solid and short-dashed lines, respectively) computed as in Gehrels (1986).

of XMMU J0338.7+0030 has a significance of $4.3 \sigma$. The significance rises to $5.3 \sigma$ if one assumes that the region external to the circular area of $2^{\prime}$ radius centred on the X-ray position of the cluster represents the coeval field environment. Considering that a value of $R_{200}$ equal to $88.8^{\prime \prime}$ (equivalent to $\sim 725 \mathrm{kpc}$ ) can be estimated from the scaling relations in Fassbender et al. (2011c), we conclude that the existence of an over-density of galaxies at the same photometric redshift of XMMU J0338.7+0030 is highly significant. This is consistent with the excess by a factor of two in the $K$ s-band galaxy number counts (for the entire region imaged with GROND) at $20.5-22.5 \mathrm{AB}$ mag with respect to several computations for the field from survey areas/deep fields (Fig. 12 in Sect. 3.1).

In addition, the projected distribution of the 44 photometric members is not concentrated towards the X-ray position of the cluster. This is consistent only in part with the original inference from the OMEGA2000 $z, H$ imaging that the 2-D distribution of the galaxies with very red $z-H$ colours is offset by about $20^{\prime \prime}$ (see Fig. 1 and Sect. 2.2.1). Indeed, the 2-D distribution of the 44 candidate cluster members in the $z^{\prime}$-image of XMMU J0338.7+0030 (right panel of Fig. 4 in Sect. 2.3.1) is broadly consistent with the weak, marginally extended X-ray emission of the cluster. A large part of the candidate members is distributed in the north-south direction. This is consistent with the location of the spectroscopic member with $\mathrm{ID}=12$, that lays $2.3^{\prime}$ away from the X-ray position of the cluster and to the north (Sect. 2.4.2). The elongation of the X-ray emission from the ICM suggests that XMMU J0338.7+0030 is still in an assembly phase.

We identify as the brightest central galaxy (BCG) the brightest photometric member, which sits on the red locus of the $i^{\prime}-z^{\prime}$ vs. $z^{\prime}$ colour-magnitude diagram of XMMU J0338.7+0030 (Fig. 15). The BCG is offset by $\sim 43^{\prime \prime}$, which corresponds to a projected distance of $\sim 350 \mathrm{kpc}$ from the X-ray position of the cluster for $z=1.1$ and the adopted cosmology (Sect. 1). This galaxy is consistently classified as elliptical by le Phare; however, it is very close to a bright star, which can affect its photometry. For this reason, the identified BCG was not selected as a target for spectroscopy with FORS2 (see Sect. 2.4.1).

Conversely, the spectroscopic member with ID $=16$ is only 2.1" away from the X-ray position of XMMU J0338.7+0030: this separation is smaller than the on-axis HEW $\left(\sim 14^{\prime \prime}\right)$ of XMM-Newton. This galaxy exhibits a weak evidence for [O II] line emission (cf. Fig. 11 and Table 1 in Sect. 2.4.2) but is identified as an E galaxy by le Phare. With $K \mathrm{~s}=21.19 \pm 0.10$ and $i^{\prime}-K \mathrm{~s}=3.36 \pm 0.12$ (i.e., $K \mathrm{~s}=19.40 \pm 0.10$ and $i^{\prime}-K \mathrm{~s}=4.77 \pm 0.12$ in the Vega magnitude system) it also meets the selection criterion of EROs (footnote 11). Its classification as a galaxy dominated by old, passively evolving stellar populations (i.e., an elliptical galaxy) or a dusty starburst is ambiguous because it exhibits $J-K \mathrm{~s}=1.28 \pm 0.13$ (i.e., $J-K \mathrm{~s}=2.17 \pm 0.13$ in the Vega magnitude system; see Pozzetti \& Mannucci 2000; see also Fig. 13 in Pierini et al. 2004a). This spectroscopic cluster member might be associated with part of the emission detected through XMM-Newton because about $15 \%$ of the $I-K$-selected EROs with $K \leq 20.1$ Vega mag exhibit $\mathrm{X}$-ray properties consistent with those expected from luminous, obscured active-galactic-nuclei (e.g., Alexander et al. 2002). If so, the true X-ray emission centroid of XMMU J0338.7+0030 might be different.

\section{Discussion}

Sections 2.1 and 2.2 have described data reduction and analysis for the archival XMM-Newton X-ray observations and the nearIR follow-up imaging with OMEGA2000 that led to the selection of XMMU J0338.7+0030 as a candidate cluster at $z>0.8$. Available FORS2 observations indicate four galaxies within 2.3' from the X-ray position of this cluster as spectroscopic members. This establishes the existence of a bound system of galaxies and hot plasma at $\langle z\rangle=1.097 \pm 0.002(1 \sigma)$ as illustrated in Figs. 10 and 11 and Table 1 (Sect. 2.4.2). With an estimated total mass $M_{200} \sim 10^{14} M_{\odot}$, XMMU J0338.7+0030 is in between X-ray selected groups and clusters (Sect. 2.1.2).

There is some tension between the spectroscopic redshift and the original estimate of $z \sim 1.45 \pm 0.15(1 \sigma)$ that was based on the tentative identification of red, passively evolving, member galaxies in the $z-H$ vs. $z$ colour-magnitude diagram of XMMU J0338.7+0030 (Fig. 3 in Sect. 2.2.2). A honest but wrong assumption (see Sect. 2.2.2) as well as systematics (see Appendix B) are at the origin of the discrepancy.

In addition to the spectroscopic observations with FORS2, we performed simultaneous optical/near-IR imaging of a region of the sky centred on XMMU J0338.7+0030 with GROND. Several pieces of evidence consistently point to the existence of an optical counterpart to the weak, marginally extended X-ray emission detected with XMM-Newton in the area imaged at all GROND $g^{\prime}, r^{\prime}, i^{\prime}, z^{\prime}, J, H, K$ s bands:

- the presence of an excess by a factor of two in the $K$ s-band galaxy number counts at $\sim 20.5-22.5 \mathrm{AB}$ mag with respect to analogous computations for the field from survey areas/deep fields (Fig. 12 in Sect. 3.1);

- the consistency between the spectroscopic redshift of the cluster and the mean value of the photometric redshifts of the three spectroscopic members imaged with GROND, which is equal to $1.12 \pm 0.09(1 \sigma)$;

- the selection of 44 sources in the photometric redshift range 1.01-1.23 that contains the three aforementioned cluster members, out of which fourteen are within a cluster-centric 


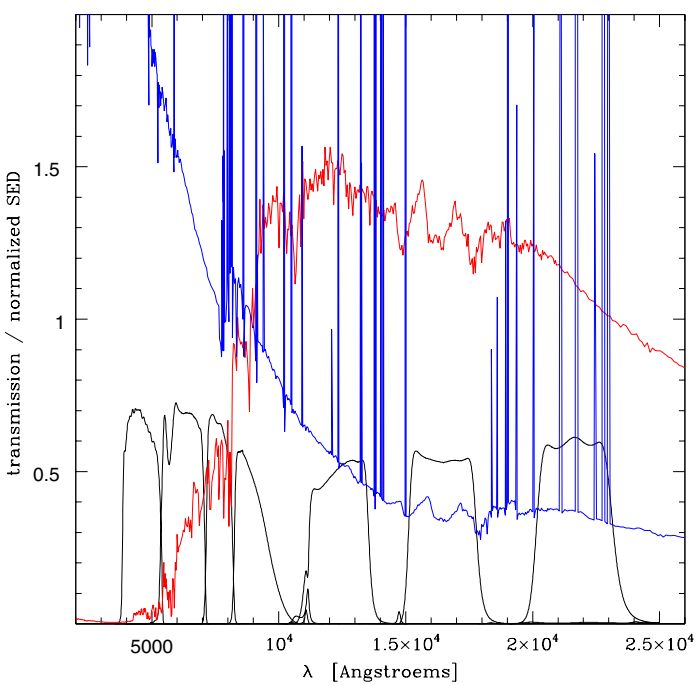

Fig. 17. Transmission curves of the GROND $g^{\prime}, r^{\prime}, i^{\prime}, z^{\prime}, J, H$, $K$ s dichroic filters (black) plus normalized SEDs that represent a galaxy dominated by an old, passively evolving stellar population (red) and a very young, dusty starburst galaxy (blue). See the text for details.

distance of 1' and signpost a galaxy over-density at a significance of $4.3 \sigma$ (Sect. 3.2.1);

- the existence of a red locus in the distribution of these 44 photo- $z$ selected galaxies in the $i^{\prime}-z^{\prime}$ vs. $z^{\prime}$ colour-magnitude diagram, which is consistent with the red sequence of the cluster RDCS J0910+5422 at $z=1.106$ (Fig. 15 in Sect. 3.2.2);

- the consistency between the projected spatial distribution of the 44 photometric cluster members and the weak, marginally extended emission of the X-ray source XMMU J0338.7+0030 (Fig. 4 in Sect. 2.3.2).

We acknowledge that the identification of three spectroscopic members out of the GROND photometric sample of 832 sources was essential to determine the photo- $z$ of the cluster as well as to select its candidate (photometric) members. Indeed, the photo- $z$ distribution of the sources within 1' from the X-ray position of the cluster exhibits two substantial excesses at photometric redshifts of $1-1.1$ and $1.2-1.3$ with respect to the photo- $z$ distribution of the sources populating the remaining area imaged by GROND at all seven bands (Fig. 14a in Sect. 3.2.1). This dicotomy is caused, at least in part, by the following reasons.

On one hand, the wavelength coverage yielded by the GROND dichroic filters for a $z=1.1$ source is not optimal. This is shown in Fig. 17, where the transmission curves of the GROND bands are reproduced together with two synthetic SEDs (from Pierini et al. 2004a) redshifted to $z=1.1$ and normalized at the effective wavelength of the $z^{\prime}$-band. One SED corresponds to an SSP model with age of 4 Gyr and solar metallicity (red); it represents a galaxy characterized by an old, passively evolving stellar population. The other corresponds to a composite stellar population model with constant star-formation rate (SFR), age of $0.1 \mathrm{Gyr}$, half-solar metallicity, attenuated by dust as for a typical nearby starburst (blue); it represents a very young, dusty starburst galaxy. The redshifted $4000 \AA$ break, a fundamental feature in redshift determination, falls in between the $i^{\prime}$-band and the $z^{\prime}$-band. The redshifted [O II] $(\lambda=3727 \AA)$ emission line falls at the red edge of the $i^{\prime}$-band, whereas the redshifted [O III $](\lambda=5007 \AA)$ and $\mathrm{H} \alpha(\lambda=6563 \AA)$ emission lines fall, respectively, in regions of the $\mathrm{z}^{\prime}$ and $J$ filters with poor transmission efficiency.
On the other hand, some ambiguity is favoured by the photometric uncertainty yielded by the present, unoptimized GROND observations, in particular at near-IR wavelengths (Fig. 7 in Sect. 2.3.2). It is known that the photometric redshift technique can be reliably extended from $z \sim 1$ to $z \sim 2$ only if deep near- and mid-IR photometry is available (e.g., Bolzonella et al. 2000; Ilbert et al. 2009).

In spite of the unfortunate mapping of the rest-frame SED of $\mathrm{a} z=1.1$ galaxy yielded by the GROND channels and the limited photometric accuracy of the present near-IR data, it is remarkable that the photometric and spectroscopic estimates of the redshift of XMMU J0338.7+0030 coincide within an uncertainty of 0.09 (cf. e.g. Lerchster et al. 2010). Indeed, the availability of spectroscopic redshifts for only $0.6 \%$ of the GROND sources (and in the narrow redshift range $0.85-1.1$ ) prevented any meaningful training of the solutions of the photo- $z$ code le Phare.

Another exquisite possibility offered by the simultaneous optical/near-IR imaging with GROND is the characterization of the galaxy population of a high- $z$ cluster on the basis of SEDs - not of a single colour-magnitude diagram - in one and the same study (cf. Mullis et al. 2005; and Lidman et al. 2008, for XMMU J2235.3-2557; Lidman et al. 2004; and Tanaka et al. 2009, for RDCS J1252.9-2927; Mei et al. 2006a; and Tanaka et al. 2008, for RDCS J0910+5422). For instance, the distribution in the $i^{\prime}-z^{\prime}$ vs. $z^{\prime}$ colour-magnitude diagram of the 44 photo- $z$ selected members of XMMU J0338.7+0030 resembles that of the members of the cluster RDCS J0910+5422 at $z=1.106$, as determined by Mei et al. (2006a), as shown in Fig. 15. At the same time, the fiducial members of XMMU J0338.7+0030 that are classified as elliptical galaxies populate a red locus in the $i^{\prime}-K$ s vs. $K$ s colour-magnitude diagram, which is broadly consistent with the red sequence of RDCS J0910+5422 determined by Tanaka et al. (2008), as shown in Fig. 18b.

The rather neat progression from red, passively evolving, elliptical galaxies to blue, star-forming, late-type spirals and irregulars in Fig. 15b is not preserved in other colour-magnitude diagrams that are used to constrain distance and properties of the galaxy population of high- $z$ clusters. The identification of a red-sequence and the classification of a cluster member galaxy as passively evolving or star forming become much more ambiguous in those colour-magnitude diagrams that involve broadband filters that do not map the redshifted $4000 \AA$ break. This is demonstrated by the distribution of the fiducial members of XMMU J0338.7+0030 in the $i^{\prime}-K$ s vs. $K$ s and $J-K$ s vs. $K$ s colour-magnitude diagrams (Figs. 18 and 19, respectively). As a reference, we reproduce the red-sequences of the spectroscopically confirmed clusters RDCS J0910+5422 at $z=1.106$ (Tanaka et al. 2008), RDCS J1252.9-2927 at $z=1.24$ (Lidman et al. 2004; Tanaka et al. 2009) and XMMU J2235.3-2557 at $z=1.39$ (Lidman et al. 2008) $^{13}$.

A galaxy classification based on either spectro-photometry or morphology indicates that there are by far not as many early-type galaxies within a cluster-centric distance of $1^{\prime}$ in XMMU J0338.7+0030 as in RDCS J0910+5422: 3 vs. 31 (Sect. 3.2.2). A high percentage of passively evolving galaxies among the brightest members of a cluster should not necessarily be expected if the cluster is observed at an early stage of its

13 The observed red-sequences and colour thresholds for $i^{\prime}-K$ s selected EROs (Pozzetti \& Mannucci 2000) and $J-K$ s selected distant red galaxies (DRGs, Franx et al. 2003) reproduced in the two figures were corrected for the different filter transmission functions, assuming a 4 Gyr-old SSP with solar metallicity (see Pierini et al. 2004a). 

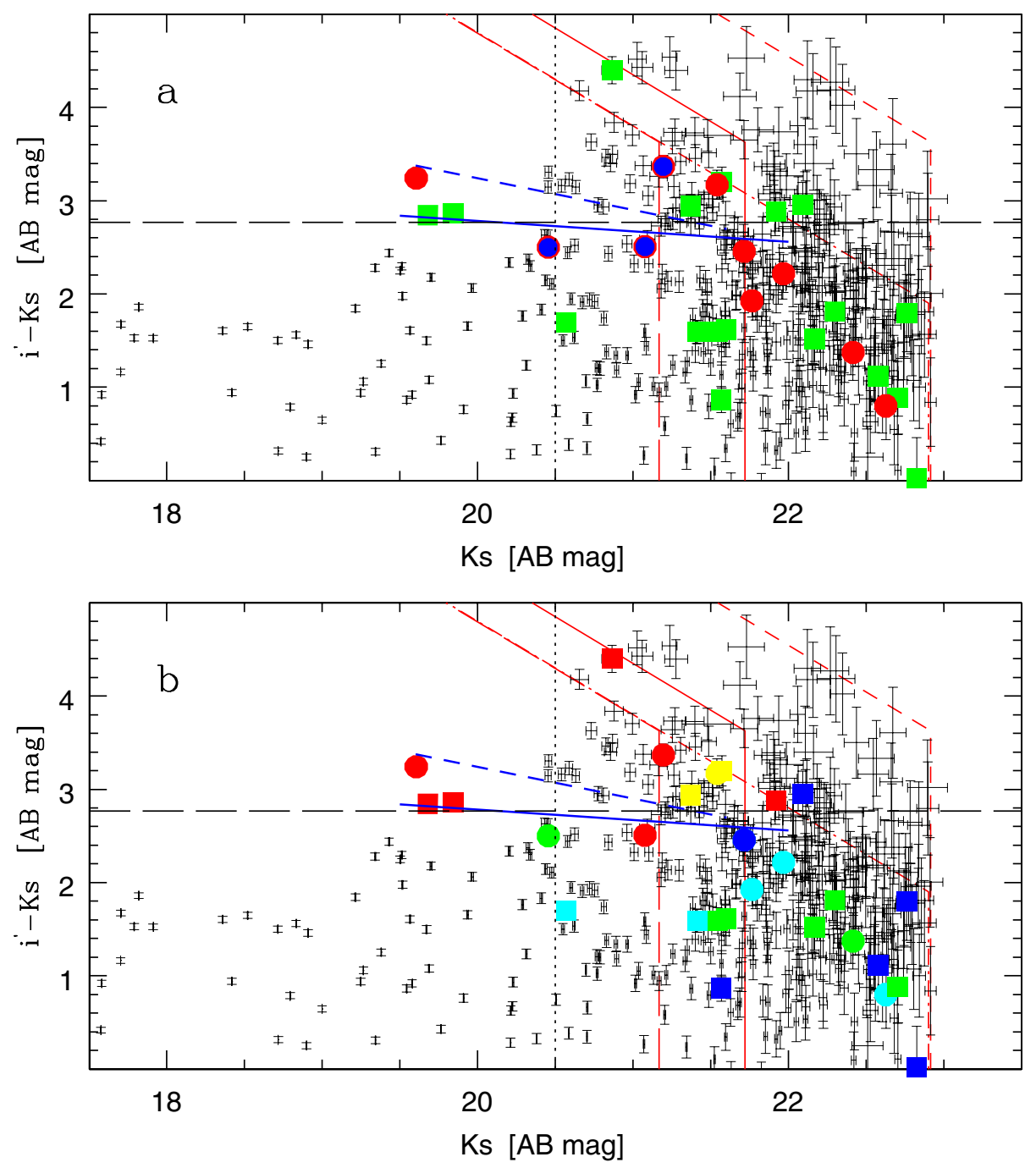

Fig. 18. $i^{\prime}-K$ s vs. $K$ s colour-magnitude diagram for all GROND sources in the XMMU J0338.7+0030 region with flux detections in the $i^{\prime}, z^{\prime}, K \mathrm{~s}$ bands at a significance level $\geq 1 \sigma$, whatever the classification of the source by Le Phare (i.e., stars are included). Photometric errors $(1 \sigma)$ are also shown. In each panel, candidate members of XMMU J0338.7+0030 are reproduced according to the corresponding panel of Fig. 15. Here the red short-dashed, solid and long-dashed lines represent the $1 \sigma, 3 \sigma$ and $5 \sigma$ flux thresholds in the $i^{\prime}, K$ s bands, respectively. The red dotted-short-dashed line represents the completeness limits in the $i^{\prime}, K$ s bands, as obtained from Fig. 12. The black vertical dotted and horizontal long-dashed lines reproduce the characteristic magnitude of the $K$ s-band luminosity function of cluster galaxies at $z \sim 1.2$ (Strazzullo et al. 2006) and the $i^{\prime}-K$ s colour-selection threshold for EROs (Pozzetti \& Mannucci 2000). As a reference, the blue solid and short-dashed lines reproduce the red sequences of the clusters RDCS J0910+5422 at $z=1.106$ (Tanaka et al. 2008) and RDCS J1252.9-2927 at $z=1.24$ (Tanaka et al. 2009), respectively. The candidate members of XMMU J0338.7+0030 that are classified as elliptical galaxies (red symbols in panel b)) populate a locus that is broadly consistent with the red sequences of the other two clusters.

halo assembly (e.g., Tanaka et al. 2008; see also Braglia et al. 2009 , for the most massive clusters at $z \sim 0.3$ ). This could be the case of XMMU J0338.7+0030, according to the projected distribution of its candidate member galaxies (Figs. 4 and 16).

The spectroscopically confirmed, optically-selected protocluster $\mathrm{Cl} 0332-2742$ at $z=1.6$ provides evidence of the buildup of the red sequence during its assembly (Kurk et al. 2009). Conversely, the X-ray luminous cluster XMMU J2235.3-2557 at $z=1.393$ (Strazzullo et al. 2010) exhibits a tight redsequence of massive galaxies, with overall old stellar populations, generally early-type morphology, typically showing earlytype spectral features and rest-frame far-UV emission consistent with very low SFRs. The massive cluster XMMU J1230.3+1339 at $z=0.975$ exhibits a richly populated red sequence, but also a few, spectro-photometrically classified, early-type galaxies that potentially host some residual star-formation activity (Lerchster et al. 2010). Consistently, the X-ray selected cluster RDCS J0910+5422, exhibits a red sequence although its early-type galaxy population appears to be still forming (Mei et al. 2006a; Tanaka et al. 2008). With a total mass of about $10^{14} M_{\odot}$ (Tanaka et al. 2008), RDCS J0910+5422 is as massive as XMMU J0338.7+0030 and four times less massive than XMMUJ1230.3+1339 at $z=0.975$ (Fassbender et al. 2011a; Lerchster et al. 2010).

If XMMU J0338.7+0030 lacks passively evolving galaxies, it hosts a high percentage of star-forming systems (late-type galaxies or starbursts), judging from the spectro-photometric classification of its candidate members (Fig. 15b). Nine of its 10 candidate members that are spectro-photometrically classified as starbursts are located well beyond a cluster-centric distance of $1^{\prime}$ (right panel of Fig. 4). Consistently, the spectroscopically confirmed member at a cluster-centric distance of $2.3^{\prime}(\mathrm{ID}=12)$ is a bright galaxy with a quite strong [O II] line emission (Sect. 2.4.2). The other bright spectroscopic member 


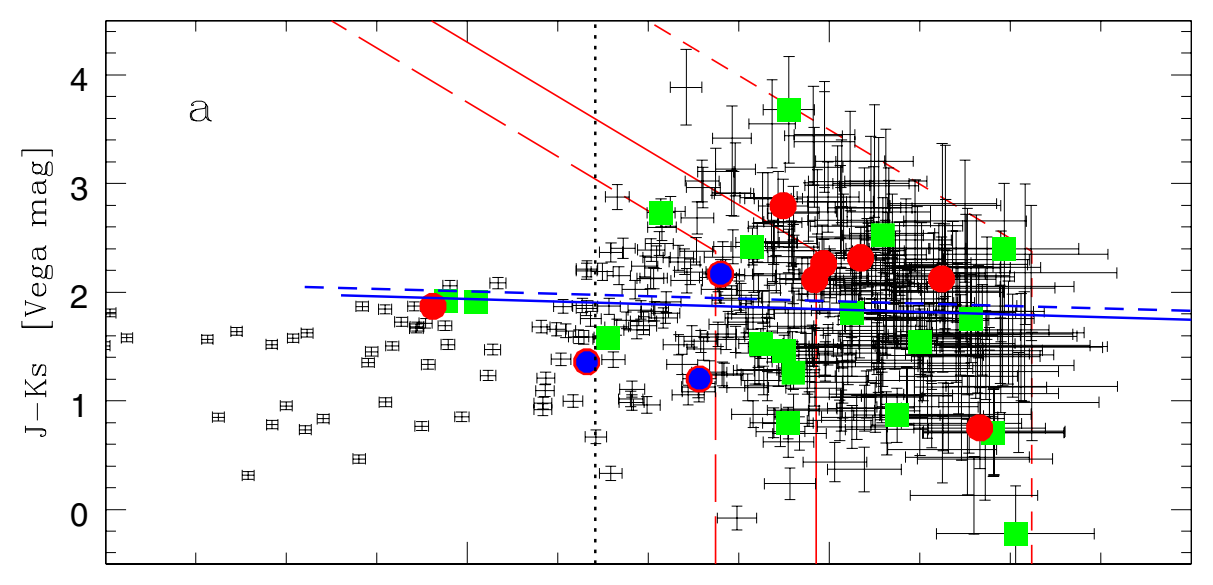

18

20

Ks [Vega mag]

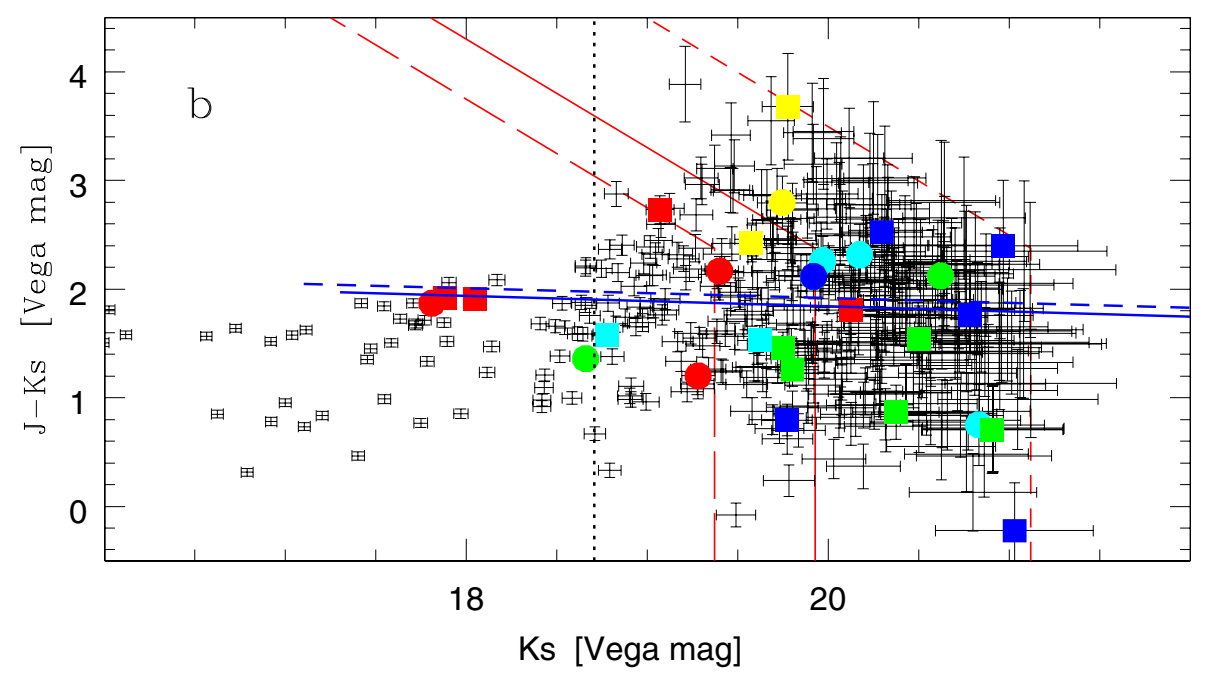

Fig. 19. $J-K$ s vs. $K$ s colour-magnitude diagram for all GROND sources in the XMMU J0338.7+0030 region with flux detections in the $z^{\prime}, J, K$ s bands at a significance level $\geq 1 \sigma$, whatever the classification of the source by Le Phare (i.e., stars are included). Photometric errors $(1 \sigma)$ are also shown. In each panel, candidate members of XMMU J0338.7+0030 are reproduced according to the corresponding panel of Fig. 15. Here the red short-dashed, solid and long-dashed lines represent the $1 \sigma, 3 \sigma$ and $5 \sigma$ flux thresholds in the $J, K$ s bands. The black vertical dotted and horizontal dotted-long-dashed lines reproduce the characteristic magnitude of the $K$ s-band luminosity function of cluster galaxies at $z \sim 1.2$ (Strazzullo et al. 2006) and the $J-K$ s colour-selection threshold for DRGs (Franx et al. 2003, see text). As a reference, the blue solid and shortdashed lines reproduce the red sequences of the clusters RDCS J1252.9-2927 at $z=1.24$ (Lidman et al. 2004) and XMMU J2235.3-2557 at $z=1.39$ (Lidman et al. 2008), respectively. The candidate members of XMMU J0338.7+0030 that are classified as elliptical galaxies (red symbols in panel b)) populate a locus that is broadly consistent with the red sequences of the other two clusters. However, candidate members that are classified as star forming span the same broad range of colours, especially at magnitudes fainter than $\sim K \mathrm{~s}^{\star}+1$.

with evidence of $[\mathrm{OII}]$ line emission $(\mathrm{ID}=15)$ is a photometric member as well but is only $50^{\prime \prime}$ away from the cluster centre and sits on the red sequence in Fig. 15 as well as the two spectroscopic members classified as elliptical galaxies $(\mathrm{ID}=2$ and $\mathrm{ID}=15)$ at cluster-centric distances of $28^{\prime \prime}$ and $2.1^{\prime \prime}$, respectively.

Interestingly, there is evidence of sustained star-formation activity at the bright end of the galaxy luminosity function in the X-ray luminous cluster XMMU J1007.4+1237 at $z=1.555$ (Fassbender et al. 2011b) and in dense environments at $z \sim 1$ (e.g., Elbaz et al. 2007; Gerke et al. 2007; Cooper et al. 2008). Several [O II] line emitters are found in the luminous, red galaxy population of XMMU J0302.2-0001 at $z=1.185$ with a total mass of a few times $10^{14} M_{\odot}$ (Šuhada et al. 2011). Spectroscopic cluster members with [O II] line emission can populate the locus of old, passively evolving galaxies or be even redder than the red sequence (see XMMU J2235.3-2557; Lidman et al. 2008).
Consistently, a non negligible fraction (20\%) of the luminous infrared galaxies in 16 optically-selected galaxy clusters at $0.4<$ $z<0.8$ sits on the corresponding red sequences (Finn et al. 2010), the remaining $80 \%$ laying below. This is no surprise when the effect of dust attenuation on the observed SED of a galaxy is computed in a physical way, tailored to its star-formation mode ${ }^{14}$ (cf. Pierini et al. 2004a,b, 2005).

In addition, star-forming galaxies at $z \sim 1-2.5$, selected in the rest-frame ultraviolet (UV) and with stellar masses of $10^{10}-10^{11} M_{\odot}$, exhibit evidence of complex configurations of stars and dusty interstellar medium (e.g., Noll et al. 2007, 2009). This points to the existence of dusty gas outflows, likely driven by feedback from supernovae, at high redshifts

14 Assumptions on the relative distribution of dust and stars as well as on the extinction law seem to impact the estimates of SFR and stellar mass in a systematic way (e.g., Küpcü Yoldaş et al. 2007). 
(cf. Steidel et al. 2010), but gravitational interactions cannot be excluded $^{15}$. Extended distributions of dust not associated with the main bodies of galaxies have been discovered in the nearby Stephan's Quintet (Natale et al. 2010) and M81 triplet of galaxies (Walter et al. 2011). Walter et al. (2011) highlight the importance of tidal stripping for the metal enrichment of the intergalactic medium at high redshifts in addition to outflows. Here we note that the additional cooling of the ICM on dust grains impacts on the X-ray scaling properties of groups and clusters of galaxies (e.g., da Silva et al. 2009; Natale et al. 2010). Furthermore, early preheating of the ICM is likely responsible for the observed evolution of the X-ray scaling relations of galaxy clusters out to $z \sim 1.5$ (Reichert et al. 2011).

In conclusion, XMMUJ0338.7+0030 appears as a bound, low-mass system of galaxies at $z=1.1$ that is caught in an early assembly phase and where the shaping of the bright end of the galaxy luminosity function and nurturing effects on galaxy evolution (see Boselli \& Gavazzi 2006, for a review) can be sought. A full understanding of the properties of the galaxy population and ICM of this cluster requires a more in-depth study based on spectroscopy and multi-wavelength imaging, which offer better statistics and/or superior quality.

\section{Conclusions}

This paper presents, in particular, results from the first pointed observations of a high- $z$ cluster with GROND, the seven-channel imager mounted at the MPI/ESO $2.2 \mathrm{~m}$ telescope of La Silla, Chile. The target was the X-ray selected, weak, marginally extended source XMMUJ0338.7+0030 in the XMM-Newton Distant Cluster Project (XDCP) survey.

Follow-up imaging with OMEGA2000 at the $3.5 \mathrm{~m}$ CAHA telescope enabled a comparison of the reddest $z-H$ colours of the galaxies within a cluster-centric distance of $45^{\prime \prime}$ with predictions from simple stellar population evolutionary models, which suggested a redshift of $z=1.45 \pm 0.15(1 \sigma)$. Later available VLT/FORS2 spectroscopy identified XMMU J0338.7+0030 as a galaxy cluster at $z=1.097 \pm 0.002(1 \sigma)$ with four spectroscopic members. From the determination of the flux in the soft $0.5-2 \mathrm{keV}$ band, the bolometric luminosity of the cluster was inferred. The ensuing X-ray estimate of the total mass of XMMU J0338.7+0030, based on a luminosity scaling relation, is equal to $M_{200} \sim 10^{14} M_{\odot}$.

About six hours of simultaneous imaging in the GROND $g^{\prime}, r^{\prime}, i^{\prime}, z^{\prime}, J, H, K \mathrm{~s}$ bands enabled us to extract photometry for 832 sources detected down to $z_{\mathrm{AB}}^{\prime} \sim 26(1 \sigma)$. Existing coverage of the same region by the shallower Sloan Digital Sky Survey and 2 Micron All Sky Survey allows a robust photometric calibration to be obtained for the optical and near-infrared (IR) channels of GROND, respectively. This is the basis for the application of the photometric redshift technique, as implemented in the publicly available code le Phare.

With the GROND data, we confirmed the existence of an optical counterpart to XMMUJ0338.7+0030 on the basis of five consistent and independent pieces of evidence. In particular, the Ks-band galaxy number counts for the cluster region imaged with GROND exhibit an excess by a factor of two at 20.5-22.5 $\mathrm{AB}$ mag with respect to those from survey

\footnotetext{
15 Hence, some complexity in the relation between bolometric infrared luminosity associated with re-emission by dust at mid-IR through submillimeter wavelengths and colour temperature of the warm and cold dust components can be expected. This could indeed have already been observed (cf. Hwang et al. 2010).
}

areas/deep fields. In addition, the photometric redshifts of the three spectroscopic members imaged with GROND (1.12 \pm 0.09 , $1 \sigma)$ is consistent with the spectroscopic redshift of the cluster. Furthermore, the distribution of the 44 candidate members of XMMU J0338.7+0030 (with photometric redshifts within the range 1.01-1.23) in the $i^{\prime}-z^{\prime}$ vs. $z^{\prime}$ colour-magnitude diagram exhibits a red locus and a bluer one that are consistent with the red sequence and the distribution of the star-forming galaxy population of the similarly massive cluster RDCS J0910+5422 at $z=1.106$.

XMMU J0338.7+0030 seems to host a galaxy population that can still undergo significant bursts of star-formation activity, as also found in other XDCP high- $z$ clusters. Part of these starforming galaxies can exhibit colours that are at least as red as those of passively evolving galaxies, owing to dust attenuation, in agreement with results in the literature. A comparison of the projected distributions of the X-ray emitting plasma and the photometric cluster members suggests that XMMU J0338.7+0030 is likely caught in an early phase of its assembly: its star-forming galaxies fall in and face an environment that rapidly changes.

Finally, we acknowledge that the identification of three spectroscopic members out of the 832 GROND sources was essential to determine the photo- $z$ of the cluster as well as to select its candidate (photometric) members. However, it is remarkable that these two estimates of the redshift of XMMU J0338.7+0030 coincide within an uncertainty of 0.09 , given that the so-called $4000 \AA$ break (a fundamental feature for photo- $z$ determination) falls in between the GROND $i^{\prime}$ and $z^{\prime}$ channels for a $z=1.1$ galaxy and the present unoptimized observations with GROND yielded a limited photometric accuracy in the near$I R$ bands. We also stress that no training of the solutions of the photo- $z$ code was performed, because the available spectroscopic information is limited to only $0.6 \%$ of the GROND sources.

For these reasons, this unique imager is useful for establishing optical counterparts to X-ray selected clusters, even in absence of spectroscopic priors. This is particularly true for high throughput surveys for clusters like the eROSITA all-sky survey, which is expected to mostly deliver massive clusters at intermediate redshifts. These clusters should host a wealthy population of old, passively evolving galaxies, which define a neat red sequence in at least one of the colour-magnitude diagrams that can be built out of the GROND optical/near-IR photometry obtained in less than two hours of observations.

Acknowledgements. D.P. thanks the anonymous referee for her/his insightful comments, which led to a signficant improvement on the robustness and presentation quality of the results in the final version of the paper. This research was supported by the DFG cluster of excellence "Origin and Structure of the Universe" (http://www . universe-cluster . de) through EXC project number 153, the DFG under grants Schw 536/24-1, Schw 536/24-2, BO 702/16-3, and the DLR under grants 50 OR 0405 and 50 QR 0802. R.S. acknowledges support by the DFG in the program SPP 1177. H.Q. thanks the FONDAP Centro de Astrofísica for partial support. F.Z. acknowledges support from and participation in the International Max-Plank Research School on Astrophysics at the Ludwig-Maximilians University. D.P. acknowledges useful feedback from O. Ilbert. D.P. acknowledges the kind hospitality at the Max-Planck-Institut für extraterrestrische Physik (MPE). This research has made use of observations collected at the European Organisation for Astronomical Research in the Southern Hemisphere (ESO), Chile (079.A-0634). This research has made use of observations collected at the Centro Astronómico Hispano Alemán (CAHA) at Calar Alto, Spain operated jointly by the Max-Planck-Institut für Astronomie and the Istituto de Astrofísica de Andalucía (CSIC). This work has made use of the SDSS database. Funding for the SDSS and SDSS-II has been provided by the Alfred P. Sloan Foundation, the Participating Institutions, the National Science Foundation, the US Department of Energy, the National Aeronautics and Space Administration, the Japanese Monbukagakusho, the Max Planck Society, and the Higher Education Funding Council for England. The SDSS Web Site is http://www.sdss.org/. The SDSS is managed by the Astrophysical Research Consortium for the Participating Institutions. The Participating 
Institutions are the American Museum of Natural History, Astrophysical Institute Potsdam, University of Basel, University of Cambridge, Case Western Reserve University, University of Chicago, Drexel University, Fermilab, the Institute for Advanced Study, the Japan Participation Group, Johns Hopkins University, the Joint Institute for Nuclear Astrophysics, the Kavli Institute for Particle Astrophysics and Cosmology, the Korean Scientist Group, the Chinese Academy of Sciences (LAMOST), Los Alamos National Laboratory, the Max-PlanckInstitute for Astronomy (MPIA), the Max-Planck-Institute for Astrophysics (MPA), New Mexico State University, Ohio State University, University of Pittsburgh, University of Portsmouth, Princeton University, the United States Naval Observatory, and the University of Washington. This publication makes use of data products from the Two Micron All Sky Survey, which is a joint project of the University of Massachusetts and the Infrared Processing and Analysis Center/California Institute of Technology, funded by the National Aeronautics and Space Administration and the National Science Foundation. This research has made use of the NASA/IPAC Extragalactic Data base (NED) which is operated by the Jet Propulsion Laboratory, California Institute of Technology, under contract with the National Aeronautics and Space Administration.

\section{References}

Abazajian, K. N., Adelman-McCarthy, J. K., Agüeros, M. A., et al. 2009, ApJS, 182,543

Albrecht, A., Bernstein, G., Cahn, R., et al. 2006, Report of the Dark Energy Task Force [arXiv: astro-ph/0609591]

Alexander, D. M., Vignali, C., Bauer, F. E., et al. 2002, AJ, 123, 1149

Appenzeller, I., Fricke, K., Fürtig, W., et al. 1998, The Messenger, 94, 1

Arimoto, N., \& Yoshii, Y. 1987, A\&A, 173, 23

Arnaboldi, M., Neeser, M. J., Parker, L. C., et al. 2007, The Messenger, 127, 28

Bailer-Jones, C. A. L., Bizenberger, P., \& Storz, C. 2000, in Optical and IR

Telescope Instrumentation and Detectors, ed. M. Iye, \& A. F. Moorwood, SPIE Proc., 4008, 1305

Balestra, I., Tozzi, P., Ettori, S., et al. 2007, A\&A, 462, 429

Bertin, E., \& Arnouts, S. 1996, A\&AS, 117, 393

Blakeslee, J. P., Franx, M., Postman, M., et al. 2003, ApJ, 596, L143

Böhringer, H., Voges, W., Huchra, J. P., et al. 2000, ApJS, 129, 435

Böhringer, H., Mullis, C., Rosati, P., et al. 2005, The Messenger, 120, 33

Bolzonella, M., Miralles, J.-M., \& Pelló, R. 2000, A\&A, 363, 476

Boselli, A., \& Gavazzi, G. 2006, PASP, 118, 517

Bower, R. G., Lucey, J. R., \& Ellis, R. S. 1992, MNRAS, 254, 601

Braglia, F. G., Pierini, D., Biviano, A., \& Böhringer, H. 2009, A\&A, 500, 947

Calzetti, D., Armus, L., Bohlin, R. C., et al. 2000, ApJ, 533, 682

Capak, P., Cowie, L. L., Hu, E. M., et al. 2004, AJ, 127, 180

Capak, P., Aussel, H., Ajiki, M., et al. 2007, ApJS, 172, 99

Cooper, M. C., Newman, J. A., Weiner, B. J., et al. 2008, MNRAS, 383, 1058

Cutri, R. M., Skrutskie, M. F., van Dyk, S., et al. 2003, The IRSA 2MASS All-

Sky Point Source Catalog, NASA/IPAC Infrared Science Archive,

http://irsa.ipac.caltech.edu/applications/Gator/

Daddi, E., Cimatti, A., Renzini, A., et al. 2004, ApJ, 617, 746

da Silva, A. C., Catalano, A., Montier, L., et al. 2009, MNRAS, 396, 849

Demarco, R., Rosati, P., Lidman, C., et al. 2007, ApJ, 663, 164

Dunkley, J., Komatsu, E., Nolta, M. R., et al. 2009, ApJS, 180, 306

Eisenhardt, P. R. M., Brodwin, M., Gonzalez, A. H., et al. 2008, ApJ, 684, 905

Elbaz, D., Daddi, E., Le Borgne, D., et al. 2007, A\&A, 468, 33

Elston, R. J., Gonzalez, A. H., McKenzie, E., et al. 2006, ApJ, 639, 816

Ettori, S., Tozzi, P., Borgani, S., \& Rosati, P. 2004, A\&A, 417, 13

Fassbender, R. 2007, Ph.D. Thesis, Ludwig-Maximilians-Universität München [arXiv:0806.0861]

Fassbender, R., Böhringer, H., Santos, J. S., et al. 2011a, A\&A, 527, A78

Fassbender, R., Nastasi, A., Böhringer, H., et al. 2011b, A\&A, 527, L10

Fassbender, R., Böhringer, H., Nastasi, A., et al. 2011c, New J. Phys., 13, 125014

Finn, R. A., Desai, V., Rudnick, G., et al. 2010, ApJ, 720, 87

Finoguenov, A., Guzzo, L., Hasinger, G., et al. 2007, ApJS, 172, 182

Fioc, M., \& Rocca-Volmerange, B. 1997, A\&A, 326, 950

Franx, M., Labbé, I., Rudnick, G., et al. 2003, ApJ, 587, L79

Galametz, A., De Breuck, C., Vernet, J., et al. 2009, A\&A, 507, 131

Gallazzi, A., Charlot, S., Brinchmann, J., White, S. D. M., \& Tremonti, C. A. 2005, MNRAS, 362, 41

Gehrels, N. 1986, ApJ, 303, 336

Gerke, B. F., Newman, J. A., Faber, S. M., et al. 2007, MNRAS, 376, 1425

Giodini, S., Pierini, D., Finoguenov, A., et al. 2009, ApJ, 703, 982

Gladders, M. D., \& Yee, H. K. C. 2000, AJ, 120, 2148

Gobat, R., Rosati, P., Strazzullo, V., et al. 2008, A\&A, 488, 853

Gobat, R., Daddi, E., Onodera, M., et al. 2011, A\&A, 526, A133

Greiner, J., Bornemann, W., Clemens, C., et al. 2008, PASP, 120, 405
Guennou, L., Adami, C., Ulmer, M. P., et al. 2010, A\&A, 523, A21

Hartley, W. G., Lane, K. P., Almaini, O., et al. 2008, MNRAS, 391, 1301

Henry, J. P., Salvato, M., Finoguenov, A., et al. 2010, ApJ, 725, 615

Hilton, M., Collins, C. A., Stanford, S. A., et al. 2007, ApJ, 670, 1000

Hilton, M., Stanford, S. A., Stott, J. P., et al. 2009, ApJ, 697, 436

Hoyle, B., Jimenez, R., \& Verde, L. 2011, Phys. Rev. D, 83, 103502

Huang, J.-S., Thompson, D., Kümmel, M. W., et al. 2001, A\&A, 368, 787

Hwang, H.-S., Elbaz, D., Magdis, G., et al. 2010, MNRAS, 409, 75

Ilbert, O., Arnouts, S., McCracken, H. J., et al. 2006, A\&A, 457, 841

Ilbert, O., Capak, P., Salvato, M., et al. 2009, ApJ, 690, 1236

Iovino, A., McCracken, H. J., Garilli, B., et al. 2005, A\&A, 442, 423

Jansen, F., Lumb, D., Altieri, B., et al. 2001, A\&A, 365, L1

Jarrett, T. H., Chester, T., Cutri, R., et al. 2000, AJ, 119, 2498

Jee, M. J., Rosati, P., Ford, H. C., et al. 2009, ApJ, 704, 672

Jordi, K., Grebel, E. K., \& Ammon, K. 2006, A\&A, 460, 339

Kaiser, N. 1984, ApJ, 284, L9

Kodama, T., Arimoto, N., Barger, A. J., \& Aragon-Salamanca, A. 1998, A\&A, 334,99

Komatsu, E., Dunkley, J., Nolta, M. R., et al. 2009, ApJS, 180, 330

Kron, R. G. 1980, ApJS, 43, 305

Krühler, T., Küpcü Yoldaş, A., Greiner, J., et al. 2008, ApJ, 685, 376

Küpcü Yoldaş, A., Salvato, M., Greiner, J., et al. 2007, A\&A, 463, 893

Küpcü Yoldaş, A., Krühler, T., Greiner, J., et al. 2008, in Gamma-Ray Bursts 2007, ed. M. Galassi, D. Palmer, \& E. Fenimore, AIP Conf. Proc., 1000, 227

Kurk, J., Cimatti, A., Zamorani, G., et al. 2009, A\&A, 504, 331

Kurtz, M. J., \& Mink, D. J. 1998, PASP, 110, 934

Lamer, G., Hoeft, M., Kohnert, J., Schwope, A. D., \& Storm, J. 2008, A\&A, 487, L33

Leauthaud, A., Massey, R., Kneib, J.-P., et al. 2007, ApJS, 172, 219

Lerchster, M., Seitz, S., Brimioulle, F., et al. 2011, MNRAS, 411, 2667

Lidman, C., Rosati, P., Demarco, R., et al. 2004, A\&A, 416, 829

Lidman, C., Rosati, P., Tanaka, M., et al. 2008, A\&A, 489, 981

Maihara, T., Iwamuro, F., Tanabe, H., et al. 2001, PASJ, 53, 25

Mantz, A., Allen, S. W., Rapetti, D., \& Ebeling, H. 2010a, MNRAS, 406, 1759

Mantz, A., Allen, S. W., Ebeling, H., Rapetti, D., \& Drlica-Wagner, A. 2010b, MNRAS, 406, 1773

Marriage, T. A., Acquaviva, V., Ade, P. A. R., et al. 2011, ApJ, 737, 61

Maughan, B. J., Jones, C., Forman, W., \& Van Speybroeck, L. 2008, ApJS, 174, 117

McCracken, H. J., Capak, P., Salvato, M., et al. 2010, ApJ, 708, 202

Mei, S., Blakeslee, J. P., Stanford, S. A., et al. 2006a, ApJ, 639, 81

Mei, S., Holden, B. P., Blakeslee, J. P., et al. 2006b, ApJ, 644, 759

Menci, N., Rosati, P., Gobat, R., et al. 2008, ApJ, 685, 863

Mullis, C. R., Rosati, P., Lamer, G., et al. 2005, ApJ, 623, L85

Nastasi, A., Fassbender, R., Böhringer, H., et al. 2011, A\&A, 532, L6

Natale, G., Tuffs, R. J., Xu, C. K., et al. 2010, ApJ, 725, 955

Noll, S., Pierini, D., Pannella, M., \& Savaglio, S. 2007, A\&A, 472, 455

Noll, S., Pierini, D., Cimatti, A., et al. 2009, A\&A, 499, 69

Oyaizu, H., Lima, M., Cunha, C. E., et al. 2008, ApJ, 674, 768

Papovich, C., Momcheva, I., Willmer, C. N. A., et al. 2010, ApJ, 716, 1503

Péroux, C., Storrie-Lombardi, L. J., McMahon, R. G., Irwin, M., Hook, I. M. 2001, AJ, 121, 1799

Petrosian, V. 1976, ApJ, 209, L1

Pierini, D., Maraston, C., Bender, R., \& Witt, A. N. 2004a, MNRAS, 347, 1

Pierini, D., Gordon, K. D., Witt, A. N., \& Madsen, G. J. 2004b, ApJ, 617, 1022

Pierini, D., Maraston, C., Gordon, K. D., \& Witt, A. N. 2005, MNRAS, 363, 131

Postman, M., Franx, M., Cross, N. J. G., et al. 2005, ApJ, 623, 721

Pozzetti, L., \& Mannucci, F. 2000, MNRAS, 317, L17

Pratt, G. W., Croston, J. H., Arnaud, M., \& Böhringer, H. 2009, A\&A, 498, 361

Prevot, M. L., Lequeux, J., Prevot, L., Maurice, E., \& Rocca-Volmerange, B. 1984, A\&A, 132, 389

Reichert, A., Böhringer, H., Fassbender, R., \& Mühlegger, M. 2011, A\&A, 535, A4

Rosati, P., della Ceca, R., Norman, C., \& Giacconi, R. 1998, ApJ, 492, L21

Rosati, P., Tozzi, P., Ettori, S., et al. 2004, AJ, 127, 230

Rosati, P., Tozzi, P., Gobat, R., et al. 2009, A\&A, 508, 583

Salpeter, E. E. 1955, ApJ, 121, 161

Santos, J. S., Rosati, P., Gobat, R., et al. 2009, A\&A, 501, 49

Santos, J. S., Tozzi, P., Rosati, P., \& Böhringer, H. 2010, A\&A, 521, A64

Santos, J. S., Fassbender, R., Nastasi, A., et al. 2011, A\&A, 531, L15

Schechter, P. 1976, ApJ, 203, 297

Schlegel, D. J., Finkbeiner, D. P., \& Davis, M. 1998, ApJ, 500, 525

Schwope, A. D., Lamer, G., de Hoon, A., et al. 2010, A\&A, 513, L10

Scodeggio, M., Franzetti, P., Garilli, B., et al. 2005, PASP, 117, 1284

Scoville, N., Aussel, H., Brusa, M., et al. 2007, ApJS, 172, 1

Skrutskie, M. F., Cutri, R. M., Stiening, R., et al. 2006, AJ, 131, 1163

Smith, J. A., Tucker, D. L., Kent, S., et al. 2002, AJ, 123, 2121

Stanford, S. A., Holden, B., Rosati, P., et al. 2001, ApJ, 552, 504 
Stanford, S. A., Holden, B., Rosati, P., et al. 2002, AJ, 123, 619

Stanford, S. A., Romer, A. K., Sabirli, K., et al. 2006, ApJ, 646, L13 Staniszewski, Z., Ade, P. A. R., Aird, K. A., et al. 2009, ApJ, 701, 32

Steidel, C. C., Erb, D. K., Shapley, A. E., et al. 2010, ApJ, 717, 289

Strazzullo, V., Rosati, P., Stanford, S. A., et al. 2006, A\&A, 450, 909

Strazzullo, V., Rosati, P., Pannella, M., et al. 2010, A\&A, 524, A17

Šuhada, R., Song, J., Böhringer, H., et al. 2010, A\&A, 514, L3

Šuhada, R., Fassbender, R., Nastasi, A., et al. 2011, A\&A, 530, A110

S̆uhada, R., Song, J., Böhringer, H., et al. 2012, A\&A, 537, A39

Sunyaev, R. A., \& Zel'dovich, Ya. B. 1972, Comm. Astrophys. Space Phys., 4, 173

Tanaka, M., Finoguenov, A., Kodama, T., et al. 2008, A\&A, 489, 571

Tanaka, M., Lidman, C., Bower, R. G., et al. 2009, A\&A, 507, 671

Tanaka, M., Finoguenov, A., \& Ueda, Y. 2010, ApJ, 716, L152

Tody, D. 1993, in Astronomical Data Analysis Software and Systems II, ed. R. J. Hanisch, R. J. V. Brissenden, \& J. Barnes, ASP Conf. Ser., 52, 173

Vanderlinde, K., Crawford, T. M., de Haan, T., et al. 2010, ApJ, 722, 1180

Vikhlinin, A., Kravtsov, A. V., Burenin, R. A., et al. 2009, ApJ, 692, 1060

Walter, F., Sandstrom, K., Aniano, G., et al. 2011, ApJ, 726, L11

White, S. D. M., \& Rees, M. J. 1978, MNRAS, 183, 341

White, S. D. M., Frenk, C. S., Davis, M., \& Efstathiou, G. 1987, ApJ, 313, 505

Wilman, D. J., Pierini, D., Tyler, K., et al. 2008, ApJ, 680, 1009

Wilson, G., Muzzin, A., Yee, H. K. C., et al. 2009, ApJ, 698, 1943

York, D. G., Adelman, J., Anderson, J. E. Jr., et al. 2000, AJ, 120, 1579

1 Max-Planck-Institut für extraterrestrische Physik (MPE), Giessenbachstrasse, 85748 Garching, Germany

e-mail: dpierini@mpe.mpg.de

2 University Observatory Munich, Scheinerstrasse 1, 81679 Munich, Germany
${ }^{3}$ Max-Planck-Institut für Plasmaphysik, Boltzmannstrasse 2, 85748 Garching, Germany

${ }^{4}$ Caltech, 1200 East California Blvd, PMA 249-17, Pasadena, CA 91125, USA

${ }^{5}$ Laboratoire AIM, IRFU/Service d'Astrophysique - CEA/DSM CNRS - Université Paris Diderot, Bât. 709, CEA-Saclay, 91191 Gifsur-Yvette Cedex, France

${ }^{6}$ European Southern Observatory (ESO), Karl-SchwarzschildStrasse 2, 85748 Garching, Germany

7 European Space Astronomy Centre (ESAC), PO Box 78, 28691 Villanueva de la Cañada, Madrid, Spain

8 Astrophysikalisches Institut Potsdam, An der Sternwarte 16, 14482 Potsdam, Germany

9 Department of Physics, Ludwig-Maximilians-Universität, Scheinerstrasse 1, 81679 Munich, Germany

10 Excellence Cluster Universe, Boltzmannstrasse 2, 85748 Garching, Germany

11 Departamento de Astronomía y Astrofísica, Pontificia Universidad Católica de Chile, Casilla 306, Santiago 22, Chile

12 Leiden Observatory, PO Box 9513, 2300 RA Leiden, The Netherlands

13 American River College, Physics and Astronomy Dept., 4700 College Oak Drive, Sacramento, CA 95841, USA

14 Dark Cosmology Centre, Niels Bohr Institute, University of Copenhagen, Juliane Maries Vej 30, 2100 Cøbenhavn Ø, Denmark

15 Institute of Astronomy, University of Cambridge, Madingley Road, Cambridge CB3 OHA, UK

16 Thüringer Landessternwarte Tautenburg, Sternwarte 5, 07778 Tautenburg, Germany 


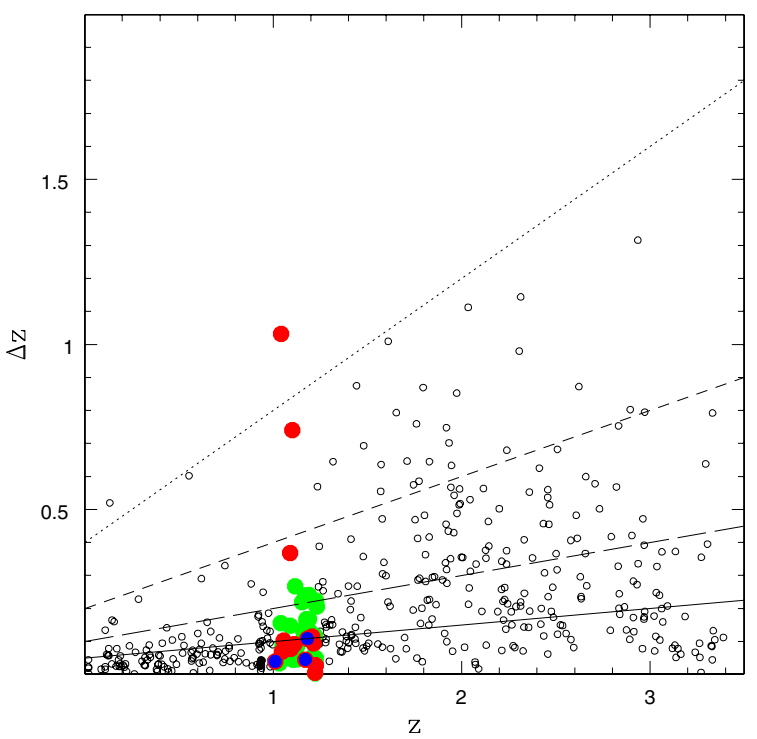

Fig. A.1. Distribution of the $68 \%$ range of the photo- $z$ solutions $(\Delta z)$ as a function of the associated best-fit photo- $z$ for the 436 GROND sources identified as galaxies (empty circles). Candidate members of XMMU J0338.7+0030 are reproduced with red (green) filled circles if they are within (outside) the bona fide cluster region; the three spectroscopic members among them are marked with blue filled circles (see Sect. 3.3.2). The dotted, short-dashed, long-dashed and solid lines represent values of $\Delta z$ equal to $0.4,0.2,0.1$ and $0.05 \times(1+z)$, respectively.

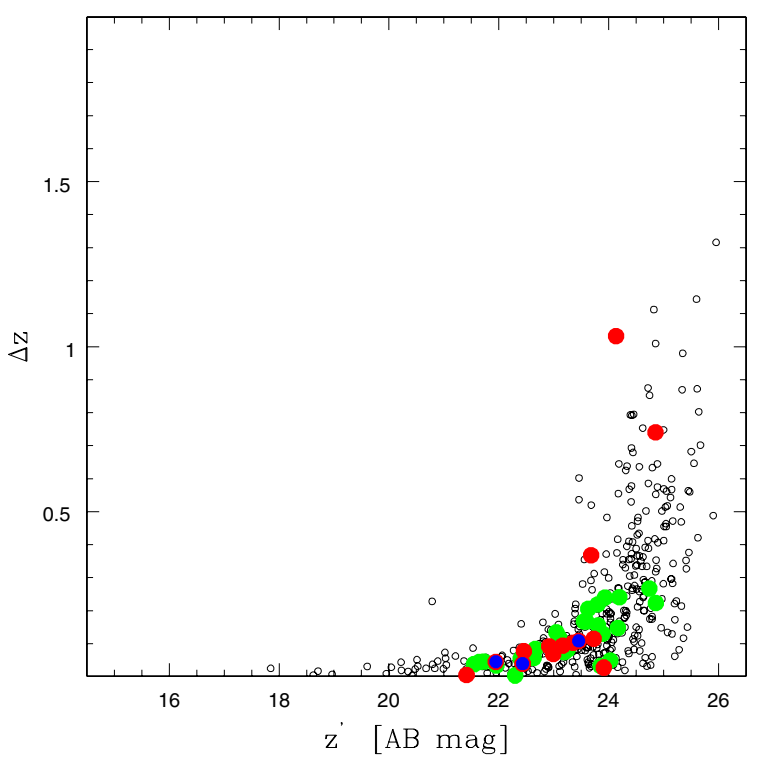

Fig. A.2. Distribution of $\Delta z$ as a function of the $z^{\prime}$-band magnitude for the same GROND sources displayed in Fig. A.1. Symbols have the same meaning as well.

\section{Appendix A: Photometric redshifts: accuracy and reliability}

Here we discuss the accuracy of the photometric estimates of the distances to the individual sources in the $3.9 \times 4.3 \mathrm{arcmin}^{2}$ region of XMMU J0338.7+0030 imaged with GROND that is achieved through the GROND multi-band photometry and the photo- $z$ code le Phare (see Sect. 3.2). At variance with works on survey areas/deep fields, we do so on the basis of photometric redshifts, mostly, because spectroscopic ones are available for only $0.6 \%$ of the GROND photometric sample (Sect. 2.4.1).

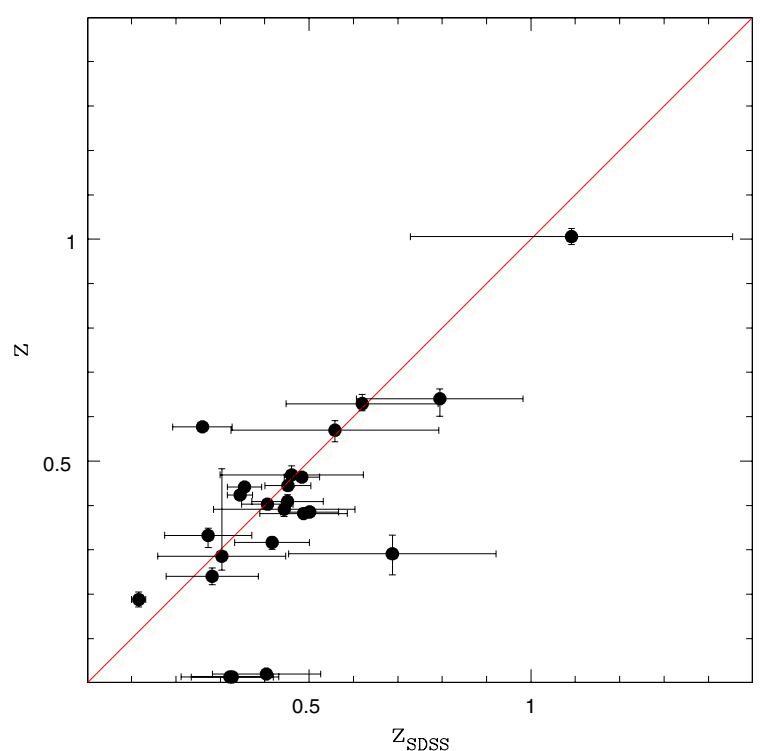

Fig. A.3. Comparison of photometric redshifts for the 24 galaxies that are in common between our GROND photometric catalogue and the SDSS DR7 one (Sect. 2.4.1). For the SDSS photo-z's, uncertainties correspond to $\pm 1 \sigma$ values. The red line represents equality.

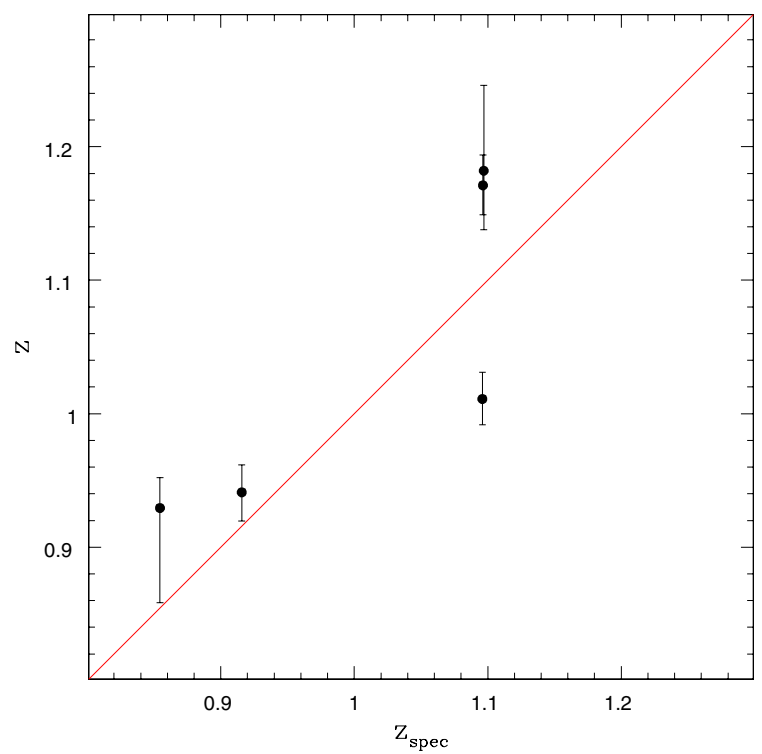

Fig. A.4. Comparison of photometric and spectroscopic redshifts for the five sources in the GROND photometric catalogue observed with FORS2 (Sect. 2.4.1). For each spectroscopic value, we show the $68 \%$ range of the photometric estimates. The red line represents equality.

In particular, we reproduce the behaviour of the $68 \%$ range of the photo- $z$ solutions yielded by le Phare, $\Delta z^{16}$, as a function of the best-fit photo- $z$ or $z^{\prime}$-band magnitude for the 436 sources out of 832 that are classified as galaxies by the best-fit solution (Figs. A.1 and A.2, respectively). The CFHTLS galaxy templates without emission lines are used. The 42 galaxies selected as fiducial cluster members (i.e., with $1.01 \leq z \leq 1.23$, see Sect. 3.3.1) are colour-coded as in Fig. 15 (Sect. 3.2.2).

Overall, the distribution of these photometric redshifts is well described by a straight line of equation $\Delta z \sim 0.05 \times(1+z)$ up to $z \sim 1.5$; only 2 photo- $z$ estimates (i.e., $0.4 \%$ ) behave as

16 Given its definition, $\Delta z$ corresponds to twice the value expected for a Gaussian photo- $z$ distribution centred at the best-fit value. 

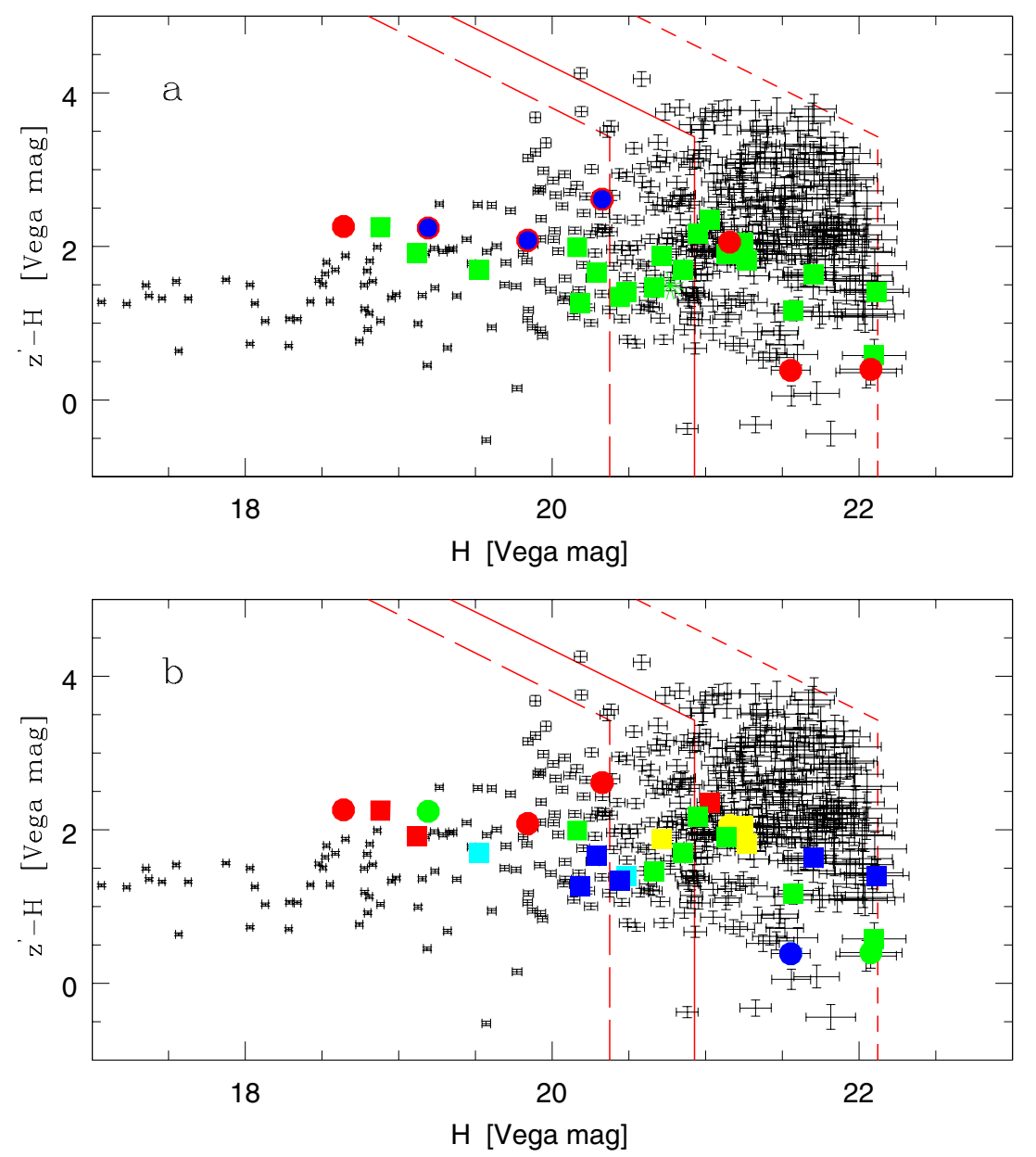

Fig. B.1. $z^{\prime}-H$ vs. $H$ colour-magnitude diagram for all GROND sources in the XMMU J0338.7+0030 region with flux detections in the $z^{\prime}, H$ bands at a significance level $\geq 1 \sigma$, whatever the classification of the source by Le Phare (i.e., stars are included). Photometric errors ( $1 \sigma)$ are also shown. As in Fig. 15, candidate members of XMMU J0338.7+0030 are reproduced with red (green) filled circles (squares) if they are within (outside) the bona fide cluster region (panel a)). The three spectroscopic cluster members in the GROND photometric catalogue are marked with blue filled circles there. Green asterisks represent the two QSO at a cluster-centric distance greater than 1'. Alternatively (panel b)), photometric cluster members classified as galaxies are colour-coded according to their spectro-photometric type, which is E (red), Sbc (yellow), Scd (green), Im (cyan) or SB (blue). In each panel, the red short-dashed, solid and long-dashed lines represent the $1 \sigma, 3 \sigma$, and $5 \sigma$ flux thresholds in the $z^{\prime}, H$ bands (cf. Sect. 2.3.2). Photometric members classified as elliptical galaxies define a narrow red locus in this colour-magnitude diagram.

outliers, in the sense that they exhibit a value of $\Delta z>0.4 \times(1+z)$. Unsurprisingly, photometric redshifts lower than 1 are assigned with an accuracy that is better than $\Delta z \sim 0.05 \times(1+z)$, whereas photometric redshifts between 1.5 and 3 exhibit a mean accuracy equal to $\Delta z \sim 0.1 \times(1+z)$ but with a large scatter. In addition, an accuracy of 0.2 or better characterizes the photometric redshifts attributed to galaxies that are brighter than $z^{\prime} \sim 23.5$, which corresponds to a $10 \sigma$ detection (see Sect. 2.3.2).

The candidate member galaxies of XMMU J0338.7+0030 tend to exhibit the lowest values of $\Delta z$ among the galaxies with the same $z^{\prime}$-band magnitude (Fig. A.2). However, three fiducial members within a cluster-centric distance of $1^{\prime}$ exhibit values of $\Delta z$ between 0.3 and 1.1: one is an outlier (Fig. A.1).

As a consistency check of our set of photometric redshifts, we compared photo-z's for 24 GROND sources with counterparts in the SDSS DR7 (see Sect. 2.3.2). The comparison SDSS photometric redshifts were calculated using a Neural Network method (Oyaizu et al. 2008). The agreement between these two independent sets of photo- $z$ estimates is good up to $z \sim 1$. As expected, our values have significantly lower uncertainties since they are based on medium-deep photometry in the $g^{\prime}, r^{\prime}, i^{\prime}, z^{\prime}, J, H, K$ s bands (e.g., Bolzonella et al. 2000).
However, the $u$-band photometry that is available from the SDSS shallower imaging in the $u, g, r, i, z$ bands (Abazajian et al. 2009 and references therein) better helps constraining photo-z's of galaxies at $z \leq 0.4$ (Bolzonella et al. 2000).

Finally, we reproduce photometric redshifts vs. spectroscopic ones for the five extragalactic sources in the GROND photometric catalogue that were observed with VLT-FORS2 (see Sect. 2.4.1) in Fig. A.4. In spite of a slight tendency towards higher values, our photometric redshifts, computed without any training of the solutions of the photo- $z$ code le Phare, agree with the spectroscopic ones within \pm 0.1 .

\section{Appendix B: On the $z^{\prime}-H$ vs. $H$ colour-magnitude diagram of XMMU J0338.7+0030}

Here we investigate the origin of the discrepancy between the spectroscopic redshift of XMMU J0338.7+0030 $(z=1.097 \pm$ 0.002 , Sect. 2.4.2) and the value that was suggested from the analysis of the $z-H$ vs. $H$ colour-magnitude diagram obtained from the CAHA imaging $(z \sim 1.45 \pm 0.15$, Sect. 2.2.2). There total magnitudes correspond to Kron aperture magnitudes i.e., computed as SExtractor MAG_AUTO (Sect. 2.1.2). 
Figure B.1 shows the $z^{\prime}-H$ vs. $H$ colour-magnitude diagram of the same cluster obtained from the GROND simultaneous multi-band imaging, where total magnitudes are computed following method $B$ (see Sect. 2.3.2). Its comparison with the analogous diagram in Fig. 3 (Sect. 2.2.2) reveals that the spectroscopic members with ID $=2$ and ID $=16$ exhibit $z^{\prime}-H$ colours that are about 0.8 Vega mag and 0.6 Vega mag bluer than their $z-H$ colours listed in Table 1 (Sect. 2.2.2). Therefore, they lie closer to the spectroscopic member with ID $=15$ (with $z^{\prime}-H=$ 2.24 Vega mag, in full agreement with its $z-H$ colour listed in Table 1) in Fig. B.1 than in Fig. 3. In addition, the existence of a red sequence is suggested in Fig. B.1. If we compute total magnitudes following method $A$, the spectroscopic members with ID $=2$ and ID $=16$ exhibit $z^{\prime}-H$ colours that are about 0.4 Vega mag bluer than their $z-H$ colours in both cases, whereas the spectroscopic member with ID $=15$ exhibits a $z^{\prime}-H$ colour that is about 0.3 Vega mag redder than its $z-H$ colour this time. The $H$-band magnitudes of these three galaxies can differ by up to \pm 0.5 Vega mag with respect to those listed in Table 1, as a function of object and method ( $A$ or $B$ ).
The method adopted to compute total magnitudes certainly impacts the estimated brightness of sources like these three galaxies, which are at the limit between being considered partly resolved or point-like for resolutions of $1.1-1.3^{\prime \prime}$. However, it is likely the stacking of individual frames without PSF-matching in the case of the OMEGA2000 images that explains the previous differences in colours.

We note that an SSP model with Salpeter IMF and solar metallicity exhibits $z-H \sim 2.4$ Vega mag when observed at $z=1.1$, for a formation redshift between 3 and 10 (Fig. 3 in Fassbender et al. 2011c). This colour is about 0.8 Vega mag bluer than the $z-H$ colour of the same model observed at $z=1.45$ (Fig. 3). It also agrees well with the distribution of the spectro-photometrically classified elliptical galaxies in Fig. B.1, modulo (small) transformations between different $z, H$ filters. At the same time, it is not inconsistent with the distribution of the spectroscopic members with absent/weak [OII] line emission $(\mathrm{ID}=2$ and $\mathrm{ID}=15)$ in Fig. 3 , taking into account the photometric uncertainties. 